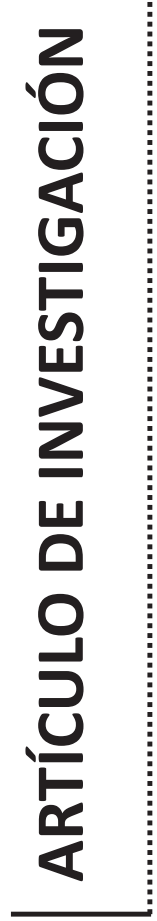




\section{Impacto del plan de vigilancia comunitaria por cuadrantes en la seguridad ciudadana del barrio Chapinero Central en Bogotá 2015-2018*} Impact of the community surveillance plan by quadrant on
citizen security in Chapinero Central neighborhood in Bogotá
2015-2018

Recibido: 02/04/2020 DOI: $10.25054 / 16576799.2515$
César Emilio Torres Reyes**

Internacionalista y magister en derecho con énfasis en derechos humanos y justicia transicional, Universidad del Rosario, Colombia

\section{RESUMEN}

El Plan Nacional de Vigilancia Comunitaria por Cuadrantes implementado desde el año 2010 en Bogotá, ha sido una estrategia operativa para mejorar el plan de acción policial ante amenazas distribuidas en zonas relativamente pequeñas. Ahora bien, la evaluación de esta política no se ha medido en términos de eficacia en la disminución del crimen en barrios. Por esta razón, se decidió hacer un análisis para evaluar el impacto de dicho plan en Chapinero Central al ser el barrio con más denuncias de hurto de Bogotá. Los resultados de esta investigación otorgan luces para entender que el plan es eficaz pero el objetivo del mismo es el que parece ceñirse a una política que no tiene en cuenta la situación que se presenta en el barrio y, por ende, los resultados no son los deseados.

\section{PALABRAS CLAVE}

Policía Reactiva; Vigilancia Comunitaria; Seguridad Ciudadana; Cuadrantes.

\section{ABSTRACT}

The National Plan for Community Surveillance by Quadrants, implemented since 2010 in Bogotá, has been an operational strategy to improve the police action plan in the face of threats distributed in relatively small areas. However, the evaluation of this policy has not been measured in terms of effectiveness in reducing crime in neighborhoods. For this reason, it was decided to make an analysis to evaluate the impact of this plan in Chapinero Central, as it is the neighborhood with the most reports of theft in Bogotá. The results of this research give light to understand that the plan is effective but the objective of it is the one that seems to stick to a policy that does not take into account the situation that occurs in the neighborhood and, therefore, the results are not the desired.

\section{KEYWORDS}

Reactive Police; Community Surveillance; Citizen Security; Quadrants

* Artículo de investigación.

** Joven investigador de la Universidad del Rosario. Investiga temas relacionados con procesos de paz, seguridad ciudadana, tecnologías y el derecho. Actualmente trabaja para la Organización Internacional para las Migraciones- ONU Migraciones. 


\section{INTRODUCCIÓN}

En américa latina el modelo de policía comunitaria fue popular, pero ineficiente en la práctica: caso bogotano.

Desde la década de los 90s, cada proclamación de reforma policial estaba relacionada con un programa dirigido a mejorar la relación entre la policía y la comunidad. Algunos de estos programas no duraron mucho, pero lo que sí es innegable es que estas estrategias en América Latina han sido bastante populares y han proclamado a lo largo de los años a través de la política, la necesidad de cumplir con las demandas de los ciudadanos (Frühling, 2012) .

Así pues, desde 1991, los esfuerzos para reformar las fuerzas policiales se multiplicaron en América Latina. Esta multiplicación estuvo motivada por la firma de los acuerdos de paz entre el gobierno y la oposición armada en El Salvador (1991) y Guatemala (1996). Los acuerdos de paz tienden a estimular la reforma de fuerzas policiales en vista de una posible finalización de un conflicto armado (Frühling, 2007) .

Si bien es cierto que en toda América Latina ha existido este interés, también es preciso decir que las adaptaciones para cumplir con el objetivo de acercar a la policía con la comunidad no se han realizado de la mejor manera y al parecer hay cierto distanciamiento entre lo que la teoría de la Policía Comunitaria dice y la práctica (Frühling, 2012) .

Para ahondar en más detalle es preciso mencionar el caso de Bogotá que fue uno de los primeros programas implementados en el continente, pero que frecuentemente ha sido citado como un ejemplo ilustrativo de la modernización de las fuerzas policiales en América Latina, sin tener en cuenta una verdadera evaluación sobre su funcionamiento (Vásquez, 2012).

Así pues, para evaluar la estrategia de policía Comunitaria en Bogotá, este artículo hará un breve recuento histórico sobre lo que se ha hecho y culminará con un análisis en la práctica sobre el último programa implementado desde 2010 denominado el Plan Nacional de Vigilancia Comunitaria por Cuadrantes (PNVCC) que ha sido desde entonces la base fundamental de la Policía Nacional de Colombia en las principales ciudades.

EL PNVCC es un bastión muy importante a la hora de establecer una policía comunitaria en cualquier ciudad, porque distribuye la labor policial en zonas geográficas relativamente pequeñas para profundizar en el acercamiento con la comunidad y en el entendimiento de problemáticas. Sin embargo, en Bogotá su implementación no ha buscado seriamente el acercamiento con la comunidad, sino más bien facilitar la labor policial operativamente.

La importancia de evaluar el impacto del PNVCC, surge en la medida que los análisis que se han hecho en Colombia sobre su eficacia se hicieron al año de su implementación y de manera muy general por parte de entidades gubernamentales. Sin embargo, no se ha hecho un estudio micro espacial a través de un barrio, para corroborar cómo se ha implementado la estrategia y qué resultados arroja en un período mayor a un año. Por lo tanto, para evaluar el impacto que ha tenido este plan operativo a través de Cuadrantes, se decidió realizar una investigación abarcando un número delimitado de cuadrantes que se encargan de implementar su programa en uno de los barrios con más problemas de hurto a personas en Bogotá: Chapinero Central.

\section{LA POLICÍA COMUNITARIA EN BOGOTÁ SE CONFORMÓ DE MANERA RIGUROSA EN LA TEORÍA, PERO EN LA PRÁCTICA EL MODELO NO SE EVALUÓ}

El modelo de Policía Comunitaria en Bogotá comenzó en 1998 cuando se establecieron contactos con la Universidad de Barcelona y L'hospitalet de Lobregat en España para que el personal experto en el tema de la "Policía de Proximidad" expusiera su plan de trabajo, metodología y resultados (Ceballos, 2004). 
Posteriormente, el presidente de la Cámara de Comercio de Bogotá, junto con el comandante de la Policía Metropolitana viajaron a España con el fin de lograr un convenio académico con la Universidad de Barcelona para lograr una capacitación para los oficiales que en su regreso se transformaría en los primeros agentes de policía comunitaria del Distrito Capital y serían los encargados de implementar el nuevo modelo (Ceballos, 2004; Vásquez, 2012).

Por lo anterior, se puede decir que el modelo de Policía Comunitaria de 1998 implementado en Bogotá tuvo muy en cuenta la teoría desde su formación pues destinaron recursos no sólo para escoger a los más idóneos para la labor de la Policía Comunitaria, sino que además los preparó académicamente y a través de experiencias internacionales.

Es así como, los requisitos para en ese entonces pertenecer al nuevo departamento de Policía Comunitaria fueron: tener una hoja de vida impecable, una predisposición para el trabajo comunitario y una recomendación de un supervisor. Los seleccionados fueron alrededor de 1064 agentes (Frühling, 2012).

Adicionalmente, el modelo policial seleccionó a 21 oficiales para que viajaran a España para familiarizarse con el modelo de vigilancia de barrios en Barcelona. Mientras que los comandantes de rango medio y bajo se educaron en la Universidad Javeriana de Bogotá para desarrollar las habilidades necesarias para acercarse a la comunidad (Frühling, 2012).

En este sentido, los oficiales que se capacitaron en España crearon el Plan de Acción de la Policía Comunitaria para Bogotá que se caracterizó por enfocarse en prevención, disuasión y servicio al cliente. Para lograr cumplir con estos tres objetivos, la Policía Comunitaria en la teoría debía tener una presencia significativa dentro de un área en particular para que los oficiales pudieran lograr una comprensión de las necesidades de la Comunidad. Sin embargo, la Cobertura no fue suficiente pues en ese entonces sólo el $8 \%$ del pie de fuerza de la Policía de Bogotá, pertenecía a la Policía Comunitaria (Frühling, 2012) .

Asimismo, a pesar de que en Colombia se implementó la Policía Comunitaria, en realidad nunca se evaluó empíricamente en el terreno. Por lo cual, no es posible afirmar que fue una estrategia exitosa, aunque se suele mencionar lo contrario (Vásquez, 2012).

Ahora bien, dicho modelo fracasó porque el modelo de Policía Comunitaria no tuvo el respaldo de todos los uniformados. Los policías encargados de la vigilancia consideraban que los compañeros de la Policía Comunitaria no ejercían bien su trabajo y, por lo tanto, se menospreciaba su labor.

Otro aspecto fundamental relacionado con el fracaso en la práctica de esta reforma policial fue que los barrios de estratos altos no tuvieron una participación en su relación comunidad-policía, porque los ciudadanos esperaban que la Policía resolviera sus problemas de convivencia y de seguridad por sí misma (Socha, 2013) .

Adicionalmente, la mayor crítica que se le hizo a este modelo fue que su desarrollo constituyó más en ofrecer un servicio adicional a la oferta policial vigente y no como una institución integral con una misión específica de trabajo. Es decir, no hubo un cambio institucional de fondo. Por lo tanto, evaluar el impacto de la implantación del modelo de la Policía comunitaria en el mejoramiento de la seguridad en Bogotá es difícil no solo por el corto tiempo de implementación, sino por otros factores como la ampliación del parque automotor (generando mayor movilidad y presencia en la calle, la creación de zonas seguras, las mejores de los servicios de CAl, la creación de frentes de seguridad local y la capacitación de los miembros de la institución) (Ceballos, 2004). 
2. LOS MODELOS DE POLICÍA NO FUNCIONAN PORQUE RESPONDEN A LA SITUACIÓN DE SEGURIDAD DEL MOMENTO: GRUPOS AL MARGEN DE LA LEY Y ACCIONES TERRORISTAS

Luego del fracaso en la práctica de la Policía Comunitaria, con el Gobierno de Álvaro Uribe la Policía retoma su anterior modelo y deja de lado el de la Policía Comunitaria. Esto se materializa con la estrategia de Seguridad Ciudadana del Período 2006-2010 que tenía como objetivo:" fortalecer la capacidad de la Policía Nacional para desarticular a los grupos y bandas responsables de la comisión de delitos de impacto en las ciudades y centro urbanos". Es decir, la nueva estrategia buscaba recuperar el orden público y combatir a los actores del conflicto armado (Socha, 2013).

En el año 2007, la Policía se enfoca en las ciudades pues en ese entonces hubo una disminución del $40 \%$ en acciones terroristas, reducción del $55 \%$ en acciones subversivas contra la Policía Nacional para el año 2007 y un aumento en la criminalidad en las principales ciudades del país, asumiendo en ese entonces una posición que, controlada la situación de los grupos armados ilegales, la Policía Nacional prevé que va a haber un tránsito de las acciones delictivas hacia el ámbito urbano (Socha, 2013).

Es así como con el Decreto 4222 del 23 de noviembre de 2006, la Dirección Operativa de la Policía Nacional comienza a denominarse Dirección de Seguridad Ciudadana y tiene como objetivo asegurar la convivencia, la seguridad y la participación ciudadana de todas las unidades del país. Ahora bien, el cambio de estructura de la Policía Nacional, a pesar de empezar con el decreto 4222, sólo se desarrolló con el Plan de Vigilancia Comunitaria por Cuadrantes que se propuso en 2010 pero que empezó a implementarse a inicios del 2011 (Socha, 2013).
Por lo cual, los modelos de policía no han funcionado porque han sido reactivos a la situación del país y, por lo tanto, nunca su implementación permanece el tiempo suficiente como para evaluar una estrategia enfocada en la seguridad ciudadana.

\section{EL FRACASO DE LA POLICÍA COMUNITARIA: VIGILANCIA COMUNITARIA}

En el año 2007 se crea la Vigilancia Comunitaria como un nuevo servicio que buscaba orientar la institución hacia lo que hoy se conoce como el Plan Nacional de Vigilancia Comunitaria por Cuadrantes, con el objetivo de lograr una reducción en los delitos de mayor impacto, mejorar la percepción de seguridad y lograr un acercamiento con el ciudadano.

Este fue el último intento de la Policía por lograr generar una cultura del servicio policial integrado con la comunidad. Se pretendía que todos los policías tuvieran características de un policía comunitario sin importar las funciones de vigilancia que estuviera desarrollando, pues el objetivo principal era acercarse a la población, mejorar la imagen institucional y recuperar la confianza (Socha, 2013).

La Policía en ese momento manifestó que no era un programa coyuntural, sino que se venía haciendo un trabajo serio y continuo en todas las unidades del país y que había mostrado frutos.

Sin embargo, los resultados de las pruebas piloto realizadas en Bogotá nunca fueron de público conocimiento y no se supo si dicho plan de vigilancia comunitaria sirvió o no. Lo que sí se sabe es que fueron en realidad muy pocos los uniformados que se dedicaron al trabajo con la población, precisamente por las exigencias de los comandantes de cada Estación para mejorar los índices delincuenciales y como tal, se dio prioridad a la necesidad de cumplir con las tareas reactivas tradicionales (Socha, 2013). 
4. EL MODELO DE POLICÍA COMUNITARIA SE DESMANTELÓ POR UN MODELO OPERATIVO, PERO SE SIGUE USANDO EL TÉRMINO "COMUNITARIO" SIN JUSTIFICACIÓN EN LA PRÁCTICA

En la retórica gubernamental se desmanteló la policía comunitaria, pero manteniendo la idea de que son cercanos a la comunidad. Sin embargo, con el nuevo Plan Nacional de Vigilancia Comunitaria por Cuadrantes (PNVCC) que desde el 2010 sigue vigente, no se tiene relación alguna con lo que significa la Policía Comunitaria. De hecho, el plan es más una cuestión operativa u organizativa de la Policía sobre el terreno.

En los últimos años, el modelo tradicional de Policía basado en la respuesta ante un hecho consumado no resultaba suficiente para responder efectivamente a la compleja situación delincuencial y las nuevas demandas de los ciudadanos en materia de seguridad; en consecuencia, la Policía evolucionó hacia un modelo operativo que iba mucho más allá de una medición netamente reactiva frente al crimen.

Es así como desde el 2010 hasta la actualidad la policía evalúa su rendimiento de acuerdo con unas metas que se estipulan en cada cuadrante, luego de haber hecho el respectivo diagnóstico de la zona. En este sentido, se evalúa la gestión policial, la reducción de crímenes y la percepción ciudadana (CCB, 2010).

Un cuadrante es una pequeña área geográfica que se encuentra delimitada para que se haga fácil el diagnóstico, intervención y monitoreo por parte de la Policía en ese lugar y a su vez se establezcan lazos estrechos con la comunidad que se encuentre en la zona, y de esta manera poder comprender sus necesidades. (Sandoval-Garrido y Marin, 2017).

Lo valioso del Plan Nacional de Vigilancia Comunitaria por Cuadrantes es que incorpora procesos para organizar el trabajo policial de manera más local y efectiva por contar con los diagnósticos locales, tablas de acción para solucionar las problemáticas y seguimiento de la gestión policial. Este modelo policial es similar al Plan Cuadrante de Seguridad Preventiva de Carabineros de Chile. Ahora bien, la estrategia en Chile hace énfasis en la relación ciudadano- policía; mientras que la estrategia de Colombia busca ofrecer un servicio policial integral en cada cuadrante, lo cual lo hace más operativo que comunitario. (OEA, 2013).

Ahora bien, es pertinente mencionar que, a pesar de llamarse Plan Nacional de Vigilancia Comunitaria, no tiene relación alguna con lo que significa una Policía Comunitaria en distintas partes del mundo. El programa, favorece la tarea operacional de la Policía y no tanto la comunitaria. El nombre tiende a confundir, pero en la práctica el plan no involucra ninguna característica de lo que en realidad sería una Policía Comunitaria como por ejemplo el vínculo estrecho con la comunidad para la prevención del crimen, la reorientación del patrullaje basado en lo que necesite la comunidad y no en órdenes jerárquicas y la rendición de cuentas (Díaz, 2011).

El Plan surgió por la necesidad de renovar el servicio de vigilancia policial porque el modelo de servicio antes del año 2009 estaba enfocado principalmente en esperar que se cometiera un crimen para desarrollar algún tipo de actividad policial. Es decir, era un modelo reactivo y establecía un plano relegado de las medidas preventivas (OEA, 2013).

Esta situación a su vez generaba un poco visibilidad del servicio social, pues su servicio estaba orientado netamente a la atención de situaciones personales de los ciudadanos y por lo tanto, realizaban pocos desplazamientos durante su servicio (OEA, 2013).

Al nivel de Estación policial, se evidenciaron que los procesos de planeación del servicio estaban enfocados en la medición de resultados acumulados, sin tener en cuenta la especificación de cada resultado para analizar una posible solución a problemáticas específicas (OEA, 2013).

En la teoría el PNVCC realiza una apreciación diagnóstica de un área geográfica determinada 
caracterizada, por recopilar información en la jurisdicción, haciendo el análisis necesario para definir los problemas que los perturban a la ciudadanía de esa zona. Es así como se determinan las causas y los factores de riesgo: tasa delictiva por 100 mil habitantes, mapa de georreferenciación delictiva y contravencional, movilidad y topografía, disponibilidad de talento humano y medios logísticos, actividad económica, grupos armados de la ley (García, 2015).

De la misma forma en cada cuadrante el objetivo es conocer e identificar el estado económico, político, cultural, delictivo y el nivel social de la zona que le corresponde a cada cuadrante (García, 2015).

Esto es posible conocerlo, en vista de que la Policía instauró en todas las estaciones de policía los Centros de Información Estratégica Policial Seccional (CIEPS) que son escenarios donde se realizan todos los estudios del fenómeno delincuencial con estadísticas, georreferenciación y posibles causas (García, 2015).

Ahora bien, si bien el PNVCC empezó formalmente en el año 2010, se hizo a modo de estrategia y con algunas pruebas piloto en las principales ciudades del país, pues sólo en enero de 2014 se elevó esta estrategia a doctrina conocida como el Modelo Nacional de Vigilancia por Comunitaria por Cuadrantes (MNVCC). Esto significa que el PNVCC al ser considerado un éxito, empezó a ser la base de lo que sería el MNVCC que sería el mismo plan, pero a modo doctrinal y con competencias no sólo en las ciudades urbanas del país, sino en municipios y áreas rurales de Colombia (García, 2015).

\section{IMPACTO DEL PLAN DE VIGILANCIA COMUNITARIA POR CUADRANTES EN EL BARRIO CHAPINERO CENTRAL DE BOGOTÁ}

\subsection{Chapinero central: el barrio más seguro en homicidios, pero más inseguro en hurto a personas}

En este sentido, no hay ningún trabajo investigativo 0 académico que demuestre cuál ha sido el impacto del Plan de Vigilancia Comunitaria por Cuadrantes en la seguridad ciudadana de un barrio y por esta razón, es que el presente artículo buscará analizar su impacto en el Barrio Chapinero Central de Bogotá. Asimismo, como el plan distribuye en zonas geográficas pequeñas el trabajo operativo de la Policía, la mejor forma de analizar su eficacia es precisamente en el barrio con el mayor índice de hurto a personas de la ciudad.

De esta manera, la investigación estará enfocada en analizar la situación de hurto a personas que se presenta en el barrio Chapinero Central, porque a pesar de ser uno de los barrios más seguros con relación al número de homicidios en Bogotá, en los últimos 5 años ha sido el barrio que más denuncias respecto al hurto a personas ha registrado.

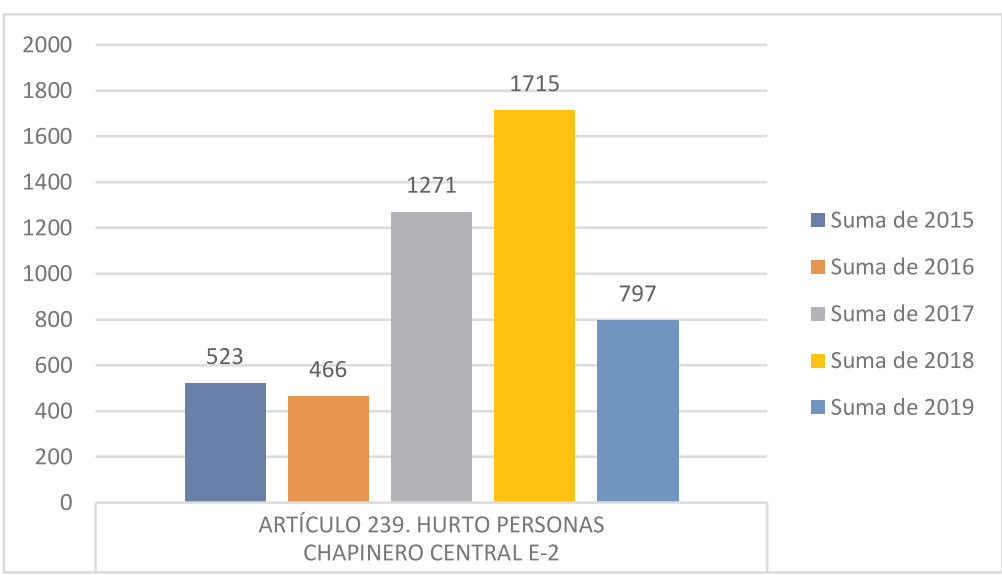

Fuente: Dijin-policía nacional. datos extraídos el día 23 de mayo del año 2019. Cifras sujetas a variación, en proceso de integración y consolidación con información de fiscalía general de la nación. 
Las cifras muestran que en el año 2017 se dio el más alto número de denuncias por hurto a personas en Chapinero Central, pero esto se debe a que en julio de 2017 se creó la plataforma virtual para que la ciudadanía reportara seis delitos específicos (hurto al comercio, residencias y personas; difusión de material con contenido de explotación sexual infantil, delitos informáticos y extorsión).

En Bogotá su funcionamiento ha sido un éxito pues cerca del $32 \%$ de las denuncias conocidas por la Fiscalía ingresaron a través del sistema virtual. Ahora bien, ese aumento dado en el año 2017 no necesariamente significa un aumento de hurto a personas, sino que se facilitó la denuncia. Sin embargo, lo que se mantiene en los últimos 5 años es que el barrio con más denuncias relacionadas con el hurto a personas es Chapinero Central (Nación, 2018).

Teniendo en cuenta que cada Cuadrante del PNVCC tiene un diagnóstico y una estrategia de mejora con respecto a la zona geográfica que le corresponde ¿Cuál es el impacto que ha tenido la estrategia del Plan de Vigilancia Comunitaria por Cuadrantes en la seguridad ciudadana de Chapinero Central entre 2015-2018? Los cuadrantes que cubren la zona de Chapinero Central son el 004 CAI CHAPINERO; 005 CAI CHAPINERO; 006 CAI CHAPINERO; 018 CAI LOURDES; 021 CAILOURDES.

\section{METODOLOGÍAYDATA}

Para evaluar el impacto, de la Policía Comunitaria y su programa de Plan de Vigilancia Comunitaria por Cuadrantes en la seguridad ciudadana de Chapinero Central en primer lugar, se recogerán los datos de los cuadrantes de la zona desde el año 2015 que son los datos más recientes que se encuentran disponibles.

Asimismo, los datos que se recolectarán serán los diagnósticos que hizo cada cuadrante de su zona geográfica y las medidas de intervención o monitoreo que se estipularon para satisfacer las necesidades de la zona. Es decir, el plan consta de unas Hojas de Registro (Diagnóstico) y de unas Tablas de Acciones Mínimas Requeridas (TAMIR) para afrontar de una mejor manera los diagnósticos de cada zona.

De esta manera, se podrá realizar un análisis crítico sobre el funcionamiento de este plan. Los diagnósticos se encuentran en la "hoja de registro" de cada cuadrante, donde se especifican las estadísticas, georreferenciación de delitos, principales problemas. Asimismo, en las TAMIR están plasmadas cada una de las acciones $u$ operativos que los oficiales realizaron durante su servicio (que suele ser de dos años). Estos datos, servirán para el propósito de esta investigación que es analizar el impacto del PNVCC en el Barrio Chapinero.

\section{LIMITACIONES}

Se pretende analizar el impacto de un programa en el Barrio Chapinero Central porque analizar toda la ciudad por cuadrantes requiere de una gran cantidad de recursos y a su vez de tiempo y disposición por parte de la Policía.

Asimismo, analizar una estrategia de cuadrantes en un barrio en específico permite un análisis más detallado sobre la eficacia del programa y comparándolo con el barrio que más denuncias recibe por hurto, permitiría analizar si las fallas en la reducción del crimen corresponden a los diagnósticos/estrategias de mejora que cada cuadrante realiza; o más bien a un problema estructural en términos de desempleo, falta de oportunidades, educación, población flotante, o la implementación de la denuncia virtual. A su vez se pretende medir la variable de hurtos con relación al PNVCC porque esta variable representa uno de los crímenes más comunes en Bogotá, y es una de las variables estándar para la medición de violencia y criminalidad. 


\section{HOJAS DE REGISTRO PLAN NACIONAL DE VIGILANCIAPOR CUADRANTES}

Las hojas de registro son estadísticas que se realizan en cada cuadrante para entender cuáles son las problemáticas con mayor afectación en cada zona. Estas hojas de registro hacen parte del Plan Nacional de Vigilancia por Cuadrantes y su fundamentación radica en que facilita atender las problemáticas en zonas relativamente pequeñas.

Adicionalmente, en las Hojas de Registro del plan si bien es cierto que manejan horarios y puntos críticos, la información suministrada para realizar esta investigación sólo muestra datos estadísticos con respecto a los casos delictivos que más se reportaron en cada uno de los cuadrantes. Por lo cual, no es claro si en las Hojas de Registro se establecen o no los puntos críticos y los horarios donde más se presentan hechos delictivos, porque en los datos suministrados no aparecen estos datos que al menos en la teoría se suponía que estaban.
Ahora bien, los horarios y los puntos críticos no son necesarios para evaluar el impacto del plan porque lo más importante en términos de seguridad ciudadana es registrar datos con relación a los delitos que más se presentan en zonas relativamente pequeñas porque de esta manera se pueden establecer estrategias para mitigarlos con la segunda parte del Plan relacionada con las TAMIR.

El material que a continuación se presentará es el original brindado por la Policía Metropolitana de Bogotá - Estación de Chapinero de Bogotá. Se decidió adjuntar los datos tal como los maneja la Policía para analizar los rubros estadísticos que se manejan en la implementación y poder apreciar si en el formato que manejan se pueden presentar dificultades o inconsistencias.

Así pues, para entender cuál es el impacto del Plan Nacional de Vigilancia por Cuadrantes en el barrio Chapinero Central de Bogotá se van a analizar las hojas de registro de los cuadrantes 04, 05, 06, 18 y 21 que sumados ocupan la totalidad del barrio desde el año 2015 hasta el 2018:

\subsection{5: los cuadrantes en conjunto reportaron el hurto a personas como la problemática más grave en Chapinero Central}

\begin{tabular}{|c|c|c|c|c|c|c|c|c|c|c|}
\hline \multicolumn{11}{|c|}{ CUADRANTE 04} \\
\hline \multirow{2}{*}{ DELICTIVO } & \multicolumn{4}{|c|}{ SEMANA 52} & \multicolumn{4}{|c|}{ ACUMULADO } & \multicolumn{2}{|c|}{ LINEA BASE 2014} \\
\hline & 2014 & 2015 & & VAR & 2014 & 2015 & & VAR & 2014 & FALTA \\
\hline HOMICIDIOS & 0 & 0 & ? & 0 & 0 & 1 & 2 & 1 & 0 & -1 \\
\hline HOMICIDIOS A/T & 0 & 0 & ! & 0 & 0 & 2 & 2 & 2 & 0 & -2 \\
\hline LESIONES COMUNES & 0 & 0 & ? & 0 & 5 & 14 & 2 & 9 & 9 & -5 \\
\hline LESIONES A/T & 0 & 0 & ! & 0 & 3 & 2 & $\vartheta$ & -1 & 4 & 2 \\
\hline HURTO VEHICULOS & 0 & 0 & 8 & 0 & 0 & 1 & $x$ & 1 & 0 & -1 \\
\hline HURTO MOTOS & 0 & 0 & ? & 0 & 1 & 0 & $\vartheta$ & -1 & 1 & 1 \\
\hline BANCOS & 0 & $\mathbf{0}$ & 8 & 0 & 0 & 0 & ? & 0 & 0 & 0 \\
\hline RESIDENCIAS & 0 & 1 & $x$ & 1 & 7 & 6 & $\vartheta$ & -1 & 10 & 4 \\
\hline PERSONAS & 3 & 0 & $\vartheta$ & -3 & 123 & 138 & 2 & 15 & 148 & 10 \\
\hline COMERCIO & 0 & 0 & 8 & 0 & 45 & 57 & $x$ & 12 & 58 & 1 \\
\hline
\end{tabular}




\begin{tabular}{|c|c|c|c|c|c|c|c|c|c|c|}
\hline \multicolumn{11}{|c|}{ CUADRANTE 05} \\
\hline \multirow{2}{*}{ DELICTIVO } & \multicolumn{4}{|c|}{ SEMANA 52} & \multicolumn{4}{|c|}{ ACUMULADO } & \multicolumn{2}{|c|}{ LINEA BASE 2014} \\
\hline & 2014 & 2015 & & VAR & 2014 & 2015 & & VAR & 2014 & FALTA \\
\hline HOMICIDIOS & 0 & $\mathbf{0}$ & 8 & 0 & 1 & 1 & p & 0 & 1 & 0 \\
\hline HOMICIDIOS A/T & 0 & $\mathbf{0}$ & 8 & 0 & 1 & 0 & 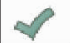 & -1 & 1 & 1 \\
\hline LESIONES COMUNES & 0 & 0 & ? & 0 & 14 & 16 & \& & 2 & 18 & 2 \\
\hline LESIONES A/T & 0 & 0 & ? & 0 & 1 & 0 & 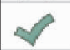 & -1 & 2 & 2 \\
\hline HURTO VEHICULOS & 0 & $\mathbf{0}$ & ? & 0 & 1 & $\mathbf{0}$ & 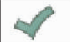 & -1 & 3 & 3 \\
\hline HURTO MOTOS & 0 & $\mathbf{0}$ & 8 & 0 & 1 & 1 & p & 0 & 1 & 0 \\
\hline BANCOS & 0 & $\mathbf{0}$ & 8 & 0 & 0 & 0 & 8 & 0 & 0 & 0 \\
\hline RESIDENCIAS & 0 & 0 & 8 & 0 & 9 & 1 & 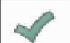 & -8 & 9 & 8 \\
\hline PERSONAS & 1 & $\mathbf{0}$ & 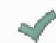 & -1 & 125 & 64 & $\leadsto$ & -61 & 149 & 85 \\
\hline COMERCIO & 0 & $\mathbf{0}$ & 8 & 0 & 27 & 19 & 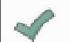 & -8 & 30 & 11 \\
\hline
\end{tabular}

\begin{tabular}{|c|c|c|c|c|c|c|c|c|c|c|}
\hline \multicolumn{11}{|c|}{ CUADRANTE 06} \\
\hline \multirow{2}{*}{ DELICTIVO } & \multicolumn{4}{|c|}{ SEMANA 52} & \multicolumn{4}{|c|}{ ACUMULADO } & \multicolumn{2}{|c|}{ LINEA BASE 2014} \\
\hline & 2014 & 2015 & & VAR & 2014 & \begin{tabular}{|l|}
2015 \\
\end{tabular} & & VAR & 2014 & FALTA \\
\hline HOMICIDIOS & 0 & 0 & 8 & 0 & $\mathbf{0}$ & $\mathbf{0}$ & 8 & 0 & 0 & 0 \\
\hline HOMICIDIOS A/T & 0 & 0 & 8 & 0 & 0 & 0 & 8 & 0 & 0 & 0 \\
\hline LESIONES COMUNES & 0 & 0 & ? & 0 & 1 & 13 & 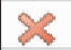 & 12 & 1 & -12 \\
\hline LESIONES A/T & 0 & 0 & $?$ & 0 & 0 & 0 & 8 & 0 & 0 & 0 \\
\hline HURTO VEHICULOS & 0 & $\mathbf{0}$ & $?$ & 0 & 0 & $\mathbf{0}$ & 8 & 0 & 0 & 0 \\
\hline HURTO MOTOS & 0 & 0 & 8 & 0 & 0 & 1 & 2 & 1 & 0 & -1 \\
\hline BANCOS & 0 & 0 & $?$ & 0 & 0 & 0 & 8 & 0 & 0 & 0 \\
\hline RESIDENCIAS & 0 & 0 & ? & 0 & 0 & 3 & $x$ & 3 & 0 & -3 \\
\hline PERSONAS & 0 & 0 & Q & 0 & 2 & 69 & $x$ & 67 & 2 & -67 \\
\hline COMERCIO & 0 & 2 & 3 & 2 & 0 & 7 & 2 & 7 & 0 & -7 \\
\hline
\end{tabular}

\begin{tabular}{|c|c|c|c|c|c|c|c|c|c|c|}
\hline \multicolumn{11}{|c|}{ CUADRANTE 18} \\
\hline \multirow{2}{*}{ DELICTIVO } & \multicolumn{4}{|c|}{ SEMANA 52} & \multicolumn{4}{|c|}{ ACUMULADO } & \multicolumn{2}{|c|}{ LINEA BASE 2014} \\
\hline & 2014 & 2015 & & VAR & 2014 & 2015 & & VAR & 2014 & FALTA \\
\hline HOMICIDIOS & $\mathbf{0}$ & 0 & ? & 0 & 0 & $\mathbf{0}$ & 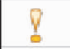 & 0 & 0 & 0 \\
\hline HOMICIDIOS A/T & 1 & $\mathbf{0}$ & 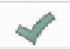 & -1 & 0 & $\mathbf{0}$ & 8 & 0 & 1 & 1 \\
\hline LESIONES COMUNES & $\mathbf{0}$ & $\mathbf{0}$ & ? & 0 & 7 & 5 & 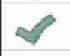 & -2 & 7 & 2 \\
\hline LESIONES A/T & 0 & 0 & ? & 0 & 2 & 11 & x & 9 & 2 & -9 \\
\hline HURTO VEHICULOS & $\mathbf{0}$ & $\mathbf{0}$ & ? & 0 & 0 & $\mathbf{0}$ & 8 & 0 & $\mathbf{0}$ & $\mathbf{0}$ \\
\hline HURTO MOTOS & 0 & 0 & ? & 0 & 2 & 2 & 8 & 0 & 2 & 0 \\
\hline BANCOS & 0 & 0 & ? & 0 & 0 & 0 & 9 & 0 & 0 & 0 \\
\hline RESIDENCIAS & $\mathbf{0}$ & $\mathbf{0}$ & ? & 0 & 2 & 2 & 8 & 0 & 4 & 2 \\
\hline PERSONAS & $\mathbf{0}$ & $\mathbf{0}$ & ? & 0 & 39 & 53 & $x$ & 14 & 47 & -6 \\
\hline COMERCIO & 0 & 0 & ? & 0 & 13 & 5 & $\because$ & -8 & 14 & 9 \\
\hline
\end{tabular}




\begin{tabular}{|c|c|c|c|c|c|c|c|c|c|c|}
\hline \multicolumn{11}{|c|}{ CUADRANTE 21} \\
\hline \multirow{2}{*}{ DELICTIVO } & \multicolumn{4}{|c|}{ SEMANA 52} & \multicolumn{4}{|c|}{ ACUMULADO } & \multicolumn{2}{|c|}{ LINEA BASE 2014} \\
\hline & 2014 & 2015 & & VAR & 2014 & 2015 & & VAR & 2014 & FALTA \\
\hline HOMICIDIOS & $\mathbf{0}$ & $\mathbf{0}$ & b & 0 & 1 & $\mathbf{0}$ & $\mathscr{F}$ & -1 & 1 & 1 \\
\hline HOMICIDIOS A/T & $\mathbf{0}$ & $\mathbf{0}$ & $!$ & 0 & $\mathbf{0}$ & 3 & 34 & 3 & $\mathbf{0}$ & -3 \\
\hline LESIONES COMUNES & $\mathbf{0}$ & $\mathbf{0}$ & b & 0 & 12 & 9 & 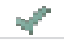 & -3 & 16 & 7 \\
\hline LESIONES A/T & $\mathbf{0}$ & $\mathbf{0}$ & 7 & 0 & 1 & $\mathbf{0}$ & 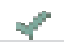 & -1 & 1 & 1 \\
\hline HURTO VEHICULOS & $\mathbf{0}$ & $\mathbf{0}$ & ! & 0 & $\mathbf{0}$ & 1 & 26 & 1 & $\mathbf{0}$ & -1 \\
\hline HURTO MOTOS & $\mathbf{0}$ & $\mathbf{0}$ & 7 & 0 & 1 & 3 & $x$ & 2 & 2 & -1 \\
\hline BANCOS & $\mathbf{0}$ & $\mathbf{0}$ & ! & 0 & $\mathbf{0}$ & 1 & 3 & 1 & $\mathbf{0}$ & -1 \\
\hline RESIDENCIAS & $\mathbf{0}$ & $\mathbf{0}$ & $?$ & 0 & 1 & 2 & 3 & 1 & 1 & -1 \\
\hline PERSONAS & 1 & $\mathbf{0}$ & r & -1 & 66 & 91 & 26 & 25 & 78 & -13 \\
\hline COMERCIO & 0 & 0 & 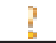 & 0 & 3 & 8 & nt & 5 & 5 & -3 \\
\hline
\end{tabular}

Tabla 1. Resumen de los cuadrantes 2015. Fuente: Hojas de registro de los cuadrantes 4, 5, 6, 18, 21 de Chapinero Central.

Durante el año 2015 en todos los cuadrantes que ocupaban la zona de Chapinero Central, presentaron como su principal problemática el hurto a personas y en segundo lugar el hurto a comercio. Lo que de alguna manera tiene relación con los datos manejados por la DIJIN en general con respecto a que en ese período fueron reportados 523 casos por hurto en el barrio, mientras que los cuadrantes reportaron 415 casos, lo que quiere decir que el plan funciona cuando se trata de tener las estadísticas en términos operacionales.

Adicionalmente, si bien es cierto que el PNVCC empezó en el año 2010, fue solamente a principios del 2011 que se empezó a implementar en toda Bogotá y se hizo de manera gradual. Por lo cual los datos desde el año 2011, 2012, 2013 y 2014 reposan en la Estación de Chapinero, pero se encuentran físicamente porque aún no han realizado el traspaso a la versión digital y en el momento de realizar esta investigación no fue posible tenerlos a disposición porque solo es posible acceder a ellos una vez la Policía Nacional realice la transferencia de dichos datos a la versión digital.

Sin embargo, para efectos de la evaluación del PNVCC es preciso decir que los datos desde el año 2015 hasta el 2018 son suficientes para entender cuál ha sido el impacto de dicho plan operacional en la seguridad ciudadana del Barrio Chapinero Central que como se ha dicho en los últimos años ha sido el principal barrio afectado por el hurto a personas en Bogotá.

\subsection{6: el hurto a personas disminuye con respecto al año anterior}

\begin{tabular}{|c|c|c|c|c|c|c|c|c|c|c|}
\hline \multicolumn{11}{|c|}{ CUADRANTE 04} \\
\hline \multirow{2}{*}{ DELICTIVO } & \multicolumn{4}{|c|}{ SEMANA 52} & \multicolumn{4}{|c|}{ ACUMULADO } & \multicolumn{2}{|c|}{ LINEA BASE 2015} \\
\hline & 2015 & 2016 & & VAR & 2015 & 2016 & & VAR & 2015 & FALTA \\
\hline HOMICIDIOS & 0 & $\mathbf{0}$ & ? & 0 & 1 & $\mathbf{0}$ & $\theta$ & -1 & 1 & 1 \\
\hline HOMICIDIOS A/T & 0 & 0 & ? & 0 & 2 & 0 & 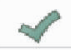 & -2 & 2 & 2 \\
\hline LESIONES COMUNES & $\mathbf{0}$ & 0 & $?$ & 0 & 14 & 6 & $\approx$ & -8 & 14 & 8 \\
\hline LESIONES A/T & 0 & 0 & ? & 0 & 2 & 0 & 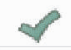 & -2 & 2 & 2 \\
\hline HURTO VEHICULOS & $\mathbf{0}$ & $\mathbf{0}$ & ? & 0 & 2 & 0 & 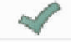 & -2 & 1 & 1 \\
\hline HURTO MOTOS & $\mathbf{0}$ & $\mathbf{0}$ & ? & 0 & $\mathbf{0}$ & 1 & 2 & 1 & $\mathbf{0}$ & -1 \\
\hline BANCOS & $\mathbf{0}$ & 0 & ? & 0 & $\mathbf{0}$ & 0 & ? & 0 & $\mathbf{0}$ & 0 \\
\hline RESIDENCIAS & 0 & 0 & 8 & 0 & 6 & 1 & $\infty$ & -5 & 6 & 5 \\
\hline PERSONAS & 1 & 2 & $x$ & 1 & 139 & 101 & $\varnothing$ & -38 & 139 & 38 \\
\hline COMERCIO & $\mathbf{0}$ & 0 & p & 0 & 57 & 4 & 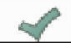 & -53 & 57 & 53 \\
\hline
\end{tabular}




\begin{tabular}{|c|c|c|c|c|c|c|c|c|c|c|}
\hline \multicolumn{11}{|c|}{ CUADRANTE 05} \\
\hline \multirow{2}{*}{ DELICTIVO } & \multicolumn{4}{|c|}{ SEMANA 52} & \multicolumn{4}{|c|}{ ACUMULADO } & \multicolumn{2}{|c|}{ LINEA BASE 2015} \\
\hline & 2015 & 2016 & & VAR & 2015 & 2016 & & VAR & 2015 & FALTA \\
\hline HOMICIDIOS & 0 & 0 & $\nabla$ & 0 & 1 & 0 & 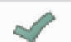 & -1 & 1 & 1 \\
\hline HOMICIDIOS A/T & $\mathbf{0}$ & 0 & $p$ & 0 & 0 & $\mathbf{0}$ & $\nabla$ & 0 & $\mathbf{0}$ & 0 \\
\hline LESIONES COMUNES & $\mathbf{0}$ & $\mathbf{0}$ & $p$ & 0 & 16 & 9 & 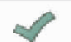 & -7 & 16 & 7 \\
\hline LESIONES A/T & 0 & 0 & $p$ & 0 & 0 & 0 & 8 & 0 & 0 & 0 \\
\hline HURTO VEHICULOS & 0 & 0 & p & 0 & 0 & 0 & $\nabla$ & 0 & 0 & 0 \\
\hline HURTO MOTOS & $\mathbf{0}$ & 0 & 8 & 0 & 1 & 7 & $x$ & 6 & 1 & -6 \\
\hline BANCOS & $\mathbf{0}$ & $\mathbf{0}$ & p & 0 & 0 & $\mathbf{0}$ & $Q$ & 0 & $\mathbf{0}$ & $\mathbf{0}$ \\
\hline RESIDENCIAS & 0 & 0 & $?$ & 0 & 1 & 4 & $2 x$ & 3 & 1 & -3 \\
\hline PERSONAS & 0 & 0 & $?$ & 0 & 65 & 79 & 2 & 14 & 65 & -14 \\
\hline COMERCIO & 0 & 0 & p & 0 & 19 & 4 & 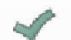 & -15 & 19 & 15 \\
\hline
\end{tabular}

\begin{tabular}{|c|c|c|c|c|c|c|c|c|c|c|}
\hline \multicolumn{11}{|c|}{ CUADRANTE 06} \\
\hline \multirow{2}{*}{ DELICTIVO } & \multicolumn{4}{|c|}{ SEMANA 52} & \multicolumn{4}{|c|}{ ACUMULADO } & \multicolumn{2}{|c|}{ LINEA BASE 2015} \\
\hline & 2015 & 2016 & & VAR & 2015 & 2016 & & VAR & 2015 & FALTA \\
\hline HOMICIDIOS & 0 & $\mathbf{0}$ & 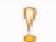 & 0 & $\mathbf{0}$ & $\mathbf{0}$ & 8 & 0 & $\mathbf{0}$ & 0 \\
\hline HOMICIDIOS A/T & 0 & 0 & 7 & 0 & 0 & 0 & 8 & 0 & 0 & 0 \\
\hline LESIONES COMUNES & $\mathbf{0}$ & $\mathbf{0}$ & 8 & 0 & 13 & 15 & 28 & 2 & 13 & -2 \\
\hline LESIONES A/T & 0 & $\mathbf{0}$ & p & 0 & $\mathbf{0}$ & $\mathbf{0}$ & 8 & 0 & 0 & 0 \\
\hline HURTO VEHICULOS & 0 & $\mathbf{0}$ & ? & 0 & $\mathbf{0}$ & 2 & 2 & 2 & $\mathbf{0}$ & -2 \\
\hline HURTO MOTOS & 0 & $\mathbf{0}$ & ? & 0 & 1 & 2 & 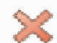 & 1 & 1 & -1 \\
\hline BANCOS & $\mathbf{0}$ & $\mathbf{0}$ & P & 0 & $\mathbf{0}$ & $\mathbf{0}$ & 8 & 0 & $\mathbf{0}$ & 0 \\
\hline RESIDENCIAS & 1 & 0 & 8 & -1 & 4 & 1 & $\infty$ & -3 & 4 & 3 \\
\hline PERSONAS & 0 & $\mathbf{0}$ & 8 & 0 & 69 & 71 & st & 2 & 69 & -2 \\
\hline COMERCIO & 0 & $\mathbf{0}$ & $p$ & 0 & 8 & 11 & se & 3 & 8 & -3 \\
\hline
\end{tabular}

\begin{tabular}{|c|c|c|c|c|c|c|c|c|c|c|}
\hline \multicolumn{11}{|c|}{ CUADRANTE 18} \\
\hline \multirow{2}{*}{ DELICTIVO } & \multicolumn{4}{|c|}{ SEMANA 52} & \multicolumn{4}{|c|}{ ACUMULADO } & \multicolumn{2}{|c|}{ LINEA BASE 2015} \\
\hline & 2015 & 2016 & & VAR & 2015 & 2016 & & VAR & 2015 & FALTA \\
\hline HOMICIDIOS & 0 & 0 & 7 & 0 & 0 & 0 & 7 & 0 & 0 & 0 \\
\hline HOMICIDIOS A/T & 0 & 0 & 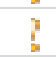 & 0 & 0 & 0 & 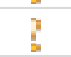 & 0 & 0 & 0 \\
\hline LESIONES COMUNES & $\mathbf{0}$ & $\mathbf{0}$ & 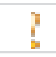 & 0 & 5 & 4 & 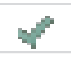 & -1 & 5 & 1 \\
\hline LESIONES A/T & 0 & 0 & 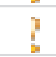 & 0 & 11 & 1 & 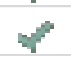 & -10 & 11 & 10 \\
\hline HURTO VEHICULOS & $\mathbf{0}$ & $\mathbf{0}$ & 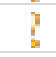 & 0 & $\mathbf{0}$ & 1 & t4 & 1 & $\mathbf{0}$ & -1 \\
\hline HURTO MOTOS & 0 & 0 & 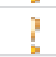 & 0 & 2 & 1 & 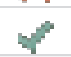 & -1 & 2 & 1 \\
\hline BANCOS & 0 & $\mathbf{0}$ & 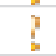 & 0 & 0 & 0 & 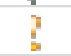 & 0 & 0 & 0 \\
\hline RESIDENCIAS & 0 & 0 & 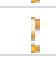 & 0 & 2 & 1 & 4 & -1 & 2 & 1 \\
\hline PERSONAS & 1 & 0 & 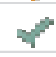 & -1 & 55 & 57 & 24 & 2 & 55 & -2 \\
\hline COMERCIO & 1 & 0 & t & -1 & 6 & 4 & $f$ & -2 & 6 & 2 \\
\hline
\end{tabular}




\begin{tabular}{|c|c|c|c|c|c|c|c|c|c|c|}
\hline \multicolumn{11}{|c|}{ CUADRANTE 21} \\
\hline DELICTIVO & 2015 & 2016 & & VAR & 2015 & 2016 & & VAR & 2015 & FALTA \\
\hline HOMICIDIOS & $\mathbf{0}$ & $\mathbf{0}$ & 7 & 0 & $\mathbf{0}$ & $\mathbf{0}$ & 7 & 0 & $\mathbf{0}$ & $\mathbf{0}$ \\
\hline HOMICIDIOS A/T & $\mathbf{0}$ & $\mathbf{0}$ & 7 & 0 & 3 & $\mathbf{0}$ & 4 & -3 & 3 & 3 \\
\hline LESIONES COMUNES & $\mathbf{0}$ & $\mathbf{0}$ & 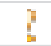 & 0 & 9 & 6 & $\Leftrightarrow$ & -3 & 9 & 3 \\
\hline LESIONES A/T & $\mathbf{0}$ & $\mathbf{0}$ & 7 & 0 & $\mathbf{0}$ & $\mathbf{0}$ & $?$ & 0 & $\mathbf{0}$ & $\mathbf{0}$ \\
\hline HURTO VEHICULOS & $\mathbf{0}$ & $\mathbf{0}$ & 7 & 0 & 3 & $\mathbf{0}$ & 4 & -3 & 1 & 1 \\
\hline HURTO MOTOS & $\mathbf{0}$ & $\mathbf{0}$ & 7 & 0 & 3 & 2 & 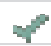 & -1 & 3 & 1 \\
\hline BANCOS & $\mathbf{0}$ & $\mathbf{0}$ & 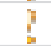 & 0 & 1 & $\mathbf{0}$ & $\Leftrightarrow$ & -1 & 1 & 1 \\
\hline RESIDENCIAS & 0 & $\mathbf{0}$ & 7 & 0 & 2 & $\mathbf{0}$ & 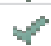 & -2 & 2 & 2 \\
\hline PERSONAS & $\mathbf{0}$ & 1 & 3 & 1 & 93 & 50 & $\%$ & -43 & 93 & 43 \\
\hline COMERCIO & $\mathbf{0}$ & 0 & $\pi$ & 0 & 8 & 6 & 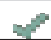 & -2 & 8 & 2 \\
\hline
\end{tabular}

Tabla 2. Resumen de los cuadrantes 2016. Fuente: Hojas de registro de los cuadrantes 4, 5, 6, 18, 21 de Chapinero Central.

En el año 2016 los casos reportados por todos los cuadrantes con relación al hurto a personas fueron de un total de 358 . Mientras que los datos reportados por la DIJIN en conjunto con respecto a los casos de hurto a personas en Chapinero Central fueron de 466 . Es decir, nuevamente existe una relación con respecto a los datos que recolecta cada cuadrante y los que maneja la Policía en general por los barrios porque tanto en los cuadrantes como en los datos en general mejoró la situación con relación a hurto a personas de 2015 a 2016.

Vale la pena mencionar que los datos reportados de los cuadrantes y de la Policía en general tienden a ser diferentes porque los cuadrantes no están distribuidos por barrios sino por zonas geográficas como se dijo anteriormente, por lo cual los cuadrantes 04, 05, 06, 18 y 21 en general ocupan geográficamente el barrio, pero también sobre pasan ligeramente esos límites con otros barrios, por lo cual es normal que los datos no sean los mismos a pesar de que tengan relación.

Adicionalmente, es claro que no todos los casos de hurto a personas sean reportados a los cuadrantes y por eso hay un mayor registro de denuncias con relación a este delito en los datos oficiales de la Policía y la DIJIN cuando publican los casos delimitados por barrios en Bogotá y no por cuadrantes.

\subsection{7: el año más problemático para los cuadrantes por la cifra más alta de casos reportados como hurto a personas en la zona}

\begin{tabular}{|c|c|c|c|c|c|c|c|c|c|c|}
\hline \multicolumn{11}{|c|}{ CUADRANTE 04} \\
\hline \multirow{2}{*}{ DELICTIVO } & \multicolumn{4}{|c|}{ SEMANA 52} & \multicolumn{4}{|c|}{ ACUM ULADO } & \multicolumn{2}{|c|}{ LINEA BASE 2016} \\
\hline & 2016 & 2017 & & VAR & 2016 & 2017 & & VAR & 2016 & FALTA \\
\hline HOMICIDIOS & $\mathbf{0}$ & $\mathbf{0}$ & 8 & 0 & $\mathbf{0}$ & 1 & $x$ & 1 & $\mathbf{0}$ & -1 \\
\hline HOMICIDIOS A/T & $\mathbf{0}$ & $\mathbf{0}$ & 8 & 0 & 1 & $\mathbf{0}$ & $\infty$ & -1 & 1 & 1 \\
\hline LESIONES COMUNES & $\mathbf{0}$ & $\mathbf{0}$ & 8 & 0 & 18 & 24 & s & 6 & 18 & -6 \\
\hline LESIONES A/T & $\mathbf{0}$ & $\mathbf{o}$ & 8 & 0 & 1 & $\mathbf{0}$ & $\curvearrowleft$ & -1 & 1 & 1 \\
\hline HURTO VEHICULOS & $\mathbf{0}$ & $\mathbf{0}$ & 8 & 0 & 1 & 2 & $x$ & 1 & 1 & -1 \\
\hline HURTO MOTOS & $\mathbf{0}$ & $\mathbf{o}$ & 8 & 0 & 6 & 5 & 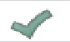 & -1 & 6 & 1 \\
\hline BANCOS & $\mathbf{0}$ & $\mathbf{0}$ & 8 & 0 & $\mathbf{0}$ & 1 & $\mathbf{x}$ & 1 & $\mathbf{0}$ & -1 \\
\hline RESIDENCIAS & $\mathbf{0}$ & $\mathbf{0}$ & 8 & 0 & 3 & 4 & s. & 1 & 3 & -1 \\
\hline PERSONAS & 2 & 1 & 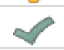 & -1 & 142 & 266 & s6 & 124 & 142 & -124 \\
\hline COMERCIO & $\mathbf{0}$ & 2 & $x$ & 2 & 18 & 64 & se & 46 & 18 & -46 \\
\hline
\end{tabular}




\begin{tabular}{|c|c|c|c|c|c|c|c|c|c|c|}
\hline \multicolumn{11}{|c|}{ CUADRANTE 05} \\
\hline \multirow{2}{*}{ DELICTIVO } & \multicolumn{4}{|c|}{ SEMANA 52} & \multicolumn{4}{|c|}{ ACUM ULADO } & \multicolumn{2}{|c|}{ LINEA BASE 2016} \\
\hline & 2016 & 2017 & & VAR & 2016 & 2017 & & VAR & 2016 & FALTA \\
\hline HOMICIDIOS & $\mathbf{0}$ & $\mathbf{0}$ & 8 & 0 & $\mathbf{0}$ & $\mathbf{0}$ & 8 & 0 & $\mathbf{0}$ & $\mathbf{0}$ \\
\hline HOMICIDIOS A/T & $\mathbf{0}$ & $\mathbf{0}$ & 8 & 0 & $\mathbf{0}$ & 1 & $\mathrm{x}$ & 1 & $\mathbf{0}$ & -1 \\
\hline LESIONES COMUNES & $\mathbf{0}$ & $\mathbf{0}$ & 8 & 0 & $\mathbf{0}$ & 1 & $x$ & 14 & $\mathbf{0}$ & -14 \\
\hline LESIONES A/T & $\mathbf{0}$ & $\mathbf{0}$ & 8 & 0 & 4 & 18 & $x$ & 2 & 4 & -1 \\
\hline HURTO VEHICULOS & 0 & 0 & 8 & 0 & 0 & 2 & 8 & 0 & 1 & 1 \\
\hline HURTO MOTOS & $\mathbf{0}$ & $\mathbf{0}$ & 8 & 0 & 0 & 0 & $\mathscr{q}$ & -1 & 1 & 1 \\
\hline BANCOS & $\mathbf{0}$ & $\mathbf{0}$ & 8 & 0 & $\mathbf{0}$ & 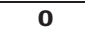 & 8 & 0 & $\mathbf{0}$ & $\mathbf{0}$ \\
\hline RESIDENCIAS & $\mathbf{0}$ & 0 & 8 & 0 & 1 & 1 & 8 & 0 & 1 & $\mathbf{0}$ \\
\hline PERSONAS & $\mathbf{0}$ & $\mathbf{0}$ & 8 & 0 & 57 & 165 & $x$ & 108 & 57 & -108 \\
\hline COMERCIO & $\mathbf{0}$ & $\mathbf{0}$ & 8 & 0 & 4 & 9 & s & 5 & 4 & -5 \\
\hline
\end{tabular}

\begin{tabular}{|c|c|c|c|c|c|c|c|c|c|c|}
\hline \multicolumn{11}{|c|}{ CUADRANTE 06} \\
\hline \multirow{2}{*}{ DELICTIVO } & \multicolumn{4}{|c|}{ SEMANA 52} & \multicolumn{4}{|c|}{ ACUM ULADO } & \multicolumn{2}{|c|}{ LINEA BASE 2016} \\
\hline & 2016 & 2017 & & VAR & 2016 & 2017 & & VAR & 2016 & FALTA \\
\hline HOMICIDIOS & $\mathbf{0}$ & $\mathbf{0}$ & 8 & 0 & $\mathbf{0}$ & $\mathbf{0}$ & 8 & 0 & $\mathbf{0}$ & $\mathbf{0}$ \\
\hline HOMICIDIOS A/T & $\mathbf{0}$ & $\mathbf{0}$ & 8 & 0 & $\mathbf{0}$ & $\mathbf{0}$ & 8 & 0 & $\mathbf{0}$ & $\mathbf{0}$ \\
\hline LESIONES COMUNES & $\mathbf{0}$ & $\mathbf{0}$ & ? & 0 & 15 & 26 & $x$ & 11 & 15 & -11 \\
\hline LESIONES A/T & $\mathbf{0}$ & $\mathbf{0}$ & จ & 0 & $\mathbf{0}$ & 0 & 8 & 0 & 0 & $\mathbf{0}$ \\
\hline HURTO VEHICULOS & 0 & 0 & 8 & 0 & 0 & $\mathbf{0}$ & 8 & 0 & 2 & 2 \\
\hline HURTO MOTOS & $\mathbf{0}$ & $\mathbf{0}$ & 8 & 0 & 2 & 2 & 8 & 0 & 2 & $\mathbf{0}$ \\
\hline BANCOS & 0 & 0 & 8 & 0 & $\mathbf{0}$ & $\mathbf{0}$ & 8 & 0 & $\mathbf{0}$ & $\mathbf{0}$ \\
\hline RESIDENCIAS & $\mathbf{0}$ & $\mathbf{0}$ & 8 & 0 & 1 & 2 & $x$ & 1 & 1 & -1 \\
\hline PERSONAS & $\mathbf{0}$ & 2 & s & 2 & 71 & 166 & $x$ & 95 & 71 & -95 \\
\hline COMERCIO & $\mathbf{0}$ & $\mathbf{0}$ & 8 & 0 & 11 & 12 & $x$ & 1 & 11 & -1 \\
\hline
\end{tabular}

\begin{tabular}{|c|c|c|c|c|c|c|c|c|c|c|}
\hline \multicolumn{11}{|c|}{ CUADRANTE 18} \\
\hline \multirow{2}{*}{ DELICTIVO } & \multicolumn{4}{|c|}{ SEMANA 52} & \multicolumn{4}{|c|}{ ACUMULADO } & \multicolumn{2}{|c|}{ LNEA BASE 2016} \\
\hline & 2016 & 2017 & & VAR & 2016 & 2017 & & VAR & 2016 & FALTA \\
\hline HOMICIDIOS & 0 & 0 & $D$ & 0 & 0 & 0 & 8 & 0 & 0 & 0 \\
\hline HOMICIDIOS A/T & 0 & o & 8 & 0 & 0 & 0 & 8 & 0 & 0 & 0 \\
\hline LESIONES COMUNES & 0 & 0 & 8 & 0 & 3 & 6 & se & 3 & 3 & -3 \\
\hline LESIONES A/T & 0 & 0 & $\nabla$ & 0 & 0 & 0 & $\nabla$ & 0 & 0 & 0 \\
\hline HURTO VEHICULOS & 0 & o & Q & 0 & 0 & 1 & 8 & 1 & 0 & -1 \\
\hline HURTO MOTOS & o & o & 8 & 0 & 1 & 0 & 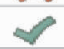 & -1 & 1 & 1 \\
\hline BANCOS & 0 & o & 8 & 0 & 0 & 0 & 8 & 0 & 0 & 0 \\
\hline RESIDENCIAS & o & $\mathbf{0}$ & ? & 0 & 3 & 3 & 8 & 0 & 3 & $\mathbf{0}$ \\
\hline PERSONAS & o & 1 & \& & 1 & 33 & 80 & s & 47 & 33 & -47 \\
\hline COMERCIO & 0 & 0 & 8 & 0 & 2 & 5 & s & 3 & 2 & -3 \\
\hline
\end{tabular}




\begin{tabular}{|c|c|c|c|c|c|c|c|c|c|c|}
\hline & & $\mathbf{C U}$ & & $\mathbf{N}$ & 21 & & & & & \\
\hline & & SEMA & 5 & & & CUMU & $\overline{\mathrm{LAD}}$ & & LINEA E & ASE 2016 \\
\hline DELIC IIVO & 2016 & 2017 & & VAR & 2016 & 2017 & & VAR & 2016 & FALTA \\
\hline HOMICIDIOS & 0 & 0 & 8 & 0 & $\mathbf{0}$ & 3 & \$8 & 3 & $\mathbf{0}$ & -3 \\
\hline HOMICIDIOS A/T & 0 & 0 & $?$ & 0 & 1 & 0 & 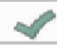 & -1 & 1 & 1 \\
\hline LESIONES COMUNES & 0 & 0 & ? & 0 & 5 & 19 & 25 & 14 & 5 & -14 \\
\hline LESIONES A/T & 0 & 0 & 8 & 0 & $\mathbf{0}$ & 4 & 25 & 4 & $\mathbf{0}$ & -4 \\
\hline HURTO VEHICULOS & 0 & 0 & 8 & 0 & 1 & 3 & S3 & 2 & $\mathbf{0}$ & -3 \\
\hline HURTO MOTOS & 0 & 0 & ? & 0 & 5 & 1 & $\sim$ & -4 & 5 & 4 \\
\hline BANCOS & 0 & 0 & ? & 0 & o & o & 8 & 0 & $\mathbf{0}$ & 0 \\
\hline RESIDENCIAS & 0 & 0 & 8 & 0 & 2 & 0 & $\infty$ & -2 & 2 & 2 \\
\hline PERSONAS & 1 & 1 & ? & 0 & 66 & 128 & se & 62 & 66 & -62 \\
\hline COMERCIO & 0 & 0 & 8 & 0 & 3 & 3 & $\nabla$ & 0 & 3 & 0 \\
\hline
\end{tabular}

Tabla 3. Resumen de los cuadrantes 2017. Fuente: Hojas de registro de los cuadrantes 4, 5, 6, 18, 21 de Chapinero Central.

Durante el año 2017 el delito más problemático fue nuevamente el hurto a personas en cada cuadrante. Sin embargo, se registró la cifra más alta en el total de cuadrantes: 805 casos de hurto a personas.

Este dato es interesante porque en el año 2017 la Secretaría de Seguridad de Bogotá explica este fenómeno afirmando que en julio de 2017 se creó la plataforma virtual ADENUNCIAR para que la ciudadanía reportara seis delitos específicos: hurto al comercio, residencias, personas; difusión de material con contenido de explotación sexual infantil, delitos informáticos y extorsión. En este sentido se creería que este aumento correspondería a la implementación de un mecanismo que facilita la denuncia.

Sin embargo, en este caso podemos inferir que realmente en el año 2017 se presenció el más alto número de casos relacionados con hurto a personas en esa zona y no debido a la implementación de la plataforma virtual porque el registro de estas estadísticas cumple una función operativa. Por lo tanto, al momento de realizar una denuncia el número de casos no estarían registrados en los cuadrantes sino en los datos que maneja la Policía Metropolitana en general.
Por ejemplo, los datos con respecto al hurto a personas en el barrio Chapinero Central para el año 2017 fueron de 1271 casos. Es decir que si bien es cierto que la aplicación facilitó la denuncia por este tipo de delito. También es preciso mencionar que el aumento no sólo se dio por la implementación de este mecanismo sino porque en ese año se registró el número más alto de casos con relación a esa modalidad de hurto tanto en los cuadrantes como en las denuncias registradas por la Policía y la Fiscalía.

Las cifras muestran que en el año 2017 se dio el más alto número de denuncias por hurto a celulares desde el 2011 en Bogotá, pero esto se debe a que en julio de 2017 se creó la plataforma virtual para que la ciudadanía reportara seis delitos específicos (hurto al comercio, residencias y personas; difusión de material con contenido de explotación sexual infantil, delitos informáticos y extorsión). En este sentido, el hurto de celulares se encuentra vinculado a la categoría de hurto a personas y por esa razón puede que se vea ese aumento en las cifras de un año a otro (Nación, 2018). 
8.4. 2018: el problema sigue siendo el mismo y en aumento. Posibles fallas en la interpretación de soluciones efectivas

\begin{tabular}{|c|c|c|c|c|c|c|c|c|c|c|}
\hline \multicolumn{11}{|c|}{ CUADRANTE 04} \\
\hline \multirow{2}{*}{ DELICTIVO } & \multicolumn{4}{|c|}{ SEM 52} & \multicolumn{4}{|c|}{ ACUMULADO } & \multicolumn{2}{|c|}{ LINEA BASE 2017} \\
\hline & 2017 & 2018 & & VAR & 2017 & 2018 & & VAR & 2017 & FALTA \\
\hline HOMICIDIOS & 0 & 0 & Q & 0 & 1 & 0 & $\approx$ & -1 & 1 & 1 \\
\hline H. BICICLETAS & 0 & 0 & p & 0 & 0 & 20 & $x$ & 20 & 0 & -20 \\
\hline LESIONES COMUNES & 0 & 0 & P & 0 & 24 & 12 & 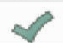 & -12 & 24 & 12 \\
\hline C. MECHERO & 1 & 3 & 2 & 2 & 38 & 126 & $x$ & 88 & $\mathbf{0}$ & -126 \\
\hline HURTO VEHICULOS & 0 & 0 & P & 0 & 0 & 1 & $x$ & 1 & 2 & 1 \\
\hline HURTO MOTOS & 0 & 0 & 8 & 0 & 5 & 4 & $\Omega$ & -1 & 5 & 1 \\
\hline BANCOS & 0 & 0 & p & 0 & 0 & 0 & 8 & 0 & 1 & 1 \\
\hline RESIDENCIAS & 0 & 0 & $p$ & 0 & 4 & 14 & $x$ & 10 & 4 & -10 \\
\hline PERSONAS & 1 & 3 & 2 & 2 & 266 & 440 & $x$ & 174 & 266 & -174 \\
\hline COMERCIO & 0 & 0 & 8 & 0 & 26 & 24 & $\rightarrow$ & -2 & 64 & 40 \\
\hline
\end{tabular}

\begin{tabular}{|c|c|c|c|c|c|c|c|c|c|c|}
\hline \multicolumn{11}{|c|}{ CUADRANTE 05} \\
\hline \multirow{2}{*}{ DELICTIVO } & \multicolumn{4}{|c|}{ SEM 52} & \multicolumn{4}{|c|}{ ACUMULADO } & \multicolumn{2}{|c|}{ LINEA BASE 2017} \\
\hline & 2017 & 2018 & & VAR & 2017 & 2018 & & VAR & 2017 & FALTA \\
\hline HOMICIDIOS & o & 0 & $p$ & 0 & 0 & 2 & 8 & 2 & 0 & -2 \\
\hline H. BICICLETAS & 0 & 0 & p & 0 & 0 & 3 & 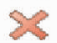 & 3 & 1 & -2 \\
\hline LESIONES COMUNES & $\mathbf{o}$ & o & D & 0 & 18 & 4 & $\approx$ & -14 & 18 & 14 \\
\hline C. MECHERO & $\mathbf{0}$ & 1 & 2 & 1 & 3 & 18 & se & 15 & 2 & -16 \\
\hline HURTO VEHICULOS & $\mathbf{0}$ & 0 & $D$ & 0 & 0 & 0 & $\nabla$ & 0 & 0 & $\mathbf{0}$ \\
\hline HURTO MOTOS & 0 & 0 & 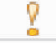 & 0 & 0 & 0 & $\nabla$ & 0 & 0 & o \\
\hline BANCOS & $\mathbf{0}$ & 0 & $p$ & 0 & 0 & 0 & $\nabla$ & 0 & 0 & 0 \\
\hline RESIDENCIAS & $\mathbf{0}$ & 0 & D & 0 & 1 & 2 & \& & 1 & 1 & -1 \\
\hline PERSONAS & 1 & 4 & 2 & 3 & 165 & 208 & s. & 43 & 165 & -43 \\
\hline COMERCIO & 0 & 0 & $P^{8}$ & 0 & 6 & 15 & $x$ & 9 & 9 & -6 \\
\hline
\end{tabular}

\begin{tabular}{|c|c|c|c|c|c|c|c|c|c|c|}
\hline \multicolumn{11}{|c|}{ CUADRANTE 06} \\
\hline \multirow{2}{*}{ DELICTIVO } & \multicolumn{4}{|c|}{ SEM 52} & \multicolumn{4}{|c|}{ ACUM ULADO } & \multicolumn{2}{|c|}{ LINEA BASE 2017} \\
\hline & 2017 & 2018 & & VAR & 2017 & 2018 & & VAR & 2017 & FALTA \\
\hline HOMICIDIOS & $\mathbf{0}$ & $\mathbf{0}$ & 8 & 0 & 0 & $\mathbf{0}$ & ? & 0 & $\mathbf{0}$ & 0 \\
\hline H. BICICLETAS & 0 & 0 & 8 & 0 & 0 & 6 & 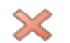 & 6 & $\mathbf{0}$ & -6 \\
\hline LESIONES COMUNES & $\mathbf{0}$ & 0 & 8 & 0 & 26 & 7 & $\checkmark$ & -19 & 26 & 19 \\
\hline C. MECHERO & $\mathbf{0}$ & $\mathbf{0}$ & 8 & 0 & 2 & 8 & ss & 6 & 0 & -8 \\
\hline HURTO VEHICULOS & o & o & $\nabla$ & 0 & $\mathbf{0}$ & 2 & 8 & 2 & $\mathbf{0}$ & -2 \\
\hline HURTO MOTOS & $\mathbf{0}$ & 0 & 8 & 0 & 2 & $\mathbf{0}$ & 8 & -2 & 2 & 2 \\
\hline BANCOS & $\mathbf{0}$ & 0 & q & 0 & 0 & 0 & ? & 0 & 0 & 0 \\
\hline RESIDENCIAS & 0 & 0 & ? & 0 & 2 & 9 & \& & 7 & 2 & -7 \\
\hline PERSONAS & o & 2 & 2x & 2 & 166 & 332 & 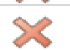 & 166 & 166 & -166 \\
\hline COMERCIO & 0 & 0 & 8 & 0 & 10 & 20 & 83 & 10 & 12 & -8 \\
\hline
\end{tabular}




\begin{tabular}{|c|c|c|c|c|c|c|c|c|c|c|}
\hline \multicolumn{11}{|c|}{ CUADRANTE 18} \\
\hline \multirow{2}{*}{ DELICTIVO } & \multicolumn{4}{|c|}{ SEM 52} & \multicolumn{4}{|c|}{ ACUMULADO } & \multicolumn{2}{|c|}{ LINEA BASE 2017} \\
\hline & 2017 & 2018 & & VAR & 2017 & 2018 & & VAR & 2017 & FALTA \\
\hline HOMICIDIOS & $\mathbf{0}$ & $\mathbf{0}$ & ? & 0 & $\mathbf{0}$ & $\mathbf{0}$ & ? & 0 & $\mathbf{0}$ & $\mathbf{0}$ \\
\hline H. BICICLETAS & 0 & 0 & 8 & 0 & 0 & 3 & $x$ & 3 & 0 & -3 \\
\hline LESIONES COMUNES & $\mathbf{0}$ & $\mathbf{0}$ & 8 & 0 & 6 & 7 & $x$ & 1 & 6 & -1 \\
\hline C. MECHERO & 0 & $\mathbf{0}$ & 8 & 0 & 3 & 20 & 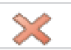 & 17 & $\mathbf{0}$ & -20 \\
\hline HURTO VEHICULOS & $\mathbf{0}$ & $\mathbf{0}$ & ? & 0 & $\mathbf{0}$ & $\mathbf{0}$ & ? & 0 & 1 & 1 \\
\hline HURTO MOTOS & $\mathbf{0}$ & $\mathbf{0}$ & 8 & 0 & $\mathbf{0}$ & 1 & 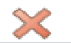 & 1 & $\mathbf{0}$ & -1 \\
\hline BANCOS & $\mathbf{0}$ & $\mathbf{0}$ & 8 & 0 & $\mathbf{0}$ & $\mathbf{0}$ & 8 & 0 & $\mathbf{0}$ & 0 \\
\hline RESIDENCIAS & $\mathbf{0}$ & $\mathbf{0}$ & ? & 0 & 3 & 7 & s & 4 & 3 & -4 \\
\hline PERSONAS & 0 & 2 & $x$ & 2 & 80 & 91 & 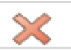 & 11 & 80 & -11 \\
\hline COMERCIO & $\mathbf{0}$ & $\mathbf{0}$ & 8 & 0 & 2 & 8 & 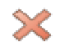 & 6 & 5 & -3 \\
\hline
\end{tabular}

\begin{tabular}{|c|c|c|c|c|c|c|c|c|c|c|}
\hline \multicolumn{11}{|c|}{ CUADRANTE 21} \\
\hline \multirow{2}{*}{ DELICTIVO } & \multicolumn{4}{|c|}{ SEM 52} & \multicolumn{4}{|c|}{ ACUM ULADO } & \multicolumn{2}{|c|}{ LINEA BASE 2017} \\
\hline & 2017 & 2018 & & VAR & 2017 & 2018 & & VAR & 2017 & FALTA \\
\hline HOMICIDIOS & 0 & 0 & 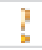 & 0 & 3 & 1 & 4 & -2 & 3 & 2 \\
\hline H. BICICLETAS & $\mathbf{0}$ & $\mathbf{0}$ & 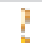 & 0 & $\mathbf{0}$ & 3 & 하 & 3 & $\mathbf{0}$ & -3 \\
\hline LESIONES COMUNES & $\mathbf{0}$ & $\mathbf{0}$ & 3 & 0 & 19 & 16 & 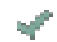 & -3 & 19 & 3 \\
\hline C. MECHERO & $\mathbf{0}$ & $\mathbf{0}$ & $?$ & 0 & $\mathbf{0}$ & 7 & th & 7 & 4 & -3 \\
\hline HURTO VEHICULOS & $\mathbf{0}$ & $\mathbf{0}$ & 7 & 0 & $\mathbf{0}$ & 3 & 3 & 3 & 3 & $\mathbf{0}$ \\
\hline HURTO MOTOS & $\mathbf{0}$ & $\mathbf{0}$ & 7 & 0 & 1 & $\mathbf{0}$ & 4 & -1 & 1 & 1 \\
\hline BANCOS & $\mathbf{0}$ & $\mathbf{0}$ & ? & 0 & $\mathbf{0}$ & $\mathbf{0}$ & 7 & 0 & 0 & 0 \\
\hline RESIDENCIAS & $\mathbf{0}$ & 0 & 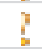 & 0 & 0 & 3 & 4 & 3 & 0 & -3 \\
\hline PERSONAS & 2 & 2 & $!$ & 0 & 128 & 300 & 3 & 172 & 128 & -172 \\
\hline COMERCIO & $\mathbf{0}$ & $\mathbf{0}$ & 2 & 0 & 3 & 7 & 3 & 4 & 3 & -4 \\
\hline
\end{tabular}

Tabla 4. Resumen de los cuadrantes 2018. Fuente: Hojas de registro de los cuadrantes 4, 5, 6, 18, 21 de Chapinero Central.

En el año 2018 la sumatoria de todos los cuadrantes registró un total de 1371 casos con relación a hurto a personas en Chapinero Central, la cifra más alta hasta el momento. Por su parte los datos de la Policía Metropolitana fueron de 1715 casos.

Las cifras a pesar de ser alarmantes reflejan a su vez que el plan, al menos con relación a las hojas de registro que realizan los cuadrantes, funciona. Cuando las cifras disminuyeron en términos generales, en los cuadrantes también disminuyó y cuando las cifras aumentaron, en los cuadrantes también aumentó. Lo que quiere decir que los cuadrantes si alcanzan a registrar problemáticas en esas zonas pequeñas en términos operacionales. Por lo tanto, la distribución geográfica a través de cuadrantes si refleja las denuncias que en general recibe la Policía Metropolitana de Bogotá.

Estas hojas de registro reflejan que en cada cuadrante los problemas de mayor impacto son: hurto de personas, lesiones comunes y hurto a comercio. Es decir que, la sumatoria de los problemas de cada cuadrante genera como conclusión que, en efecto, Chapinero Central es una de las zonas más afectadas por el hurto de personas.

Así pues, es posible determinar que las hojas de registro del Plan Nacional de Vigilancia por Cuadrantes funcionan puesto que las estadísticas de cada cuadrante señalan que el principal problema de la zona es el hurto a personas, así como lo hacen las 
estadísticas que reflejan que el barrio donde suelen presentarse más casos de hurto a personas es Chapinero Central.

\section{TAMIR (TABLAS DE ACCIONES MÍNIMAS REQUERIDAS)}

Son las tablas que llevan el registro de los operativos realizados por cada cuadrante en su respectiva zona. En este sentido, el objetivo del Plan es básicamente lograr que con las hojas de registro se realice un diagnóstico y con las TAMIR realizar operativos encaminados a disminuir las principales problemáticas bien sabidas por los diagnósticos realizados por cada cuadrante.
En las Hojas de Registro se argumentó en este artículo que el plan en ese aspecto funcionaba porque los datos de los cuadrantes tenían una relación con los datos que manejaba la Policía Metropolitana para esa zona. Es decir que, en términos estadísticos los cuadrantes si funcionan para reflejar una problemática en un sector operacional pequeño.

Dicho lo anterior, a continuación, se presentarán las tablas TAMIR de los cuadrantes que ocupan la totalidad del Barrio Chapinero Central para luego analizar si llevan alguna relación con las hojas de registro mencionadas anteriormente:

\subsection{5: operativos enfocados en estupefacientes ¿existe relación entre el crimen y las drogas?}

\begin{tabular}{|c|c|c|c|c|c|c|c|c|c|c|}
\hline \multicolumn{11}{|c|}{ CUADRANTE 04} \\
\hline \multirow{2}{*}{ OPERATIVO } & \multicolumn{4}{|c|}{ SEMANA 52} & \multicolumn{4}{|c|}{ ACUMULADO } & \multicolumn{2}{|c|}{ LINEABASE 2014} \\
\hline & 2014 & 2015 & & VAR & 2014 & 2015 & & VAR & 2014 & FALTA \\
\hline MERCANCIA RECUPERADA & 1 & 0 & \multirow{2}{*}{8} & -1 & 51 & 75 & $\leadsto$ & 24 & 55 & 20 \\
\hline VEHICULOS RECUPERADO & 0 & 0 & & 0 & 0 & 0 & ? & 0 & 0 & 0 \\
\hline MOTOS RECUPERADA & 0 & 0 & ? & 0 & 0 & 0 & ? & 0 & 0 & 0 \\
\hline ESTUPEFACIENTES & 0 & 0 & ? & 0 & 642 & 75 & \& & -567 & 642 & -567 \\
\hline CAPTURAS FLAGRANCIA & 2 & 0 & $2 \times$ & -2 & 65 & 99 & 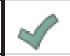 & 34 & 69 & 30 \\
\hline POR ORDEN JUDICIAL & 0 & 0 & ? & 0 & 4 & 0 & $x$ & -4 & 4 & -4 \\
\hline POR PORTE ILEGAL & 0 & 0 & ? & 0 & 0 & 0 & ? & 0 & 1 & -1 \\
\hline POR DECRETO & 0 & 0 & 7 & 0 & 1 & 0 & 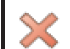 & -1 & 1 & -1 \\
\hline
\end{tabular}

\begin{tabular}{|c|c|c|c|c|c|c|c|c|c|c|}
\hline \multicolumn{11}{|c|}{ CUADRANTE 05} \\
\hline \multirow{2}{*}{ OPERATIVO } & \multicolumn{4}{|c|}{ SEMANA 52} & \multicolumn{4}{|c|}{ ACUMULADO } & \multicolumn{2}{|c|}{ LINEA BASE 2014} \\
\hline & 2014 & 2015 & & VAR & 2014 & 2015 & & VAR & 2014 & FALTA \\
\hline MERCANCIA RECUPERADA & 0 & 0 & ? & 0 & 28 & 22 & 2 & -6 & 28 & -6 \\
\hline VEHICULOS RECUPERADO & 0 & 0 & 8 & 0 & 0 & 0 & 8 & 0 & 0 & 0 \\
\hline MOTOS RECUPERADA & 0 & 0 & 8 & 0 & 0 & 6 & $\mathscr{V}$ & 6 & 0 & 6 \\
\hline ESTUPEFACIENTES & 0 & 0 & 8 & 0 & 3581 & 6477 & 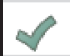 & 2896 & 3581 & 2896 \\
\hline CAPTURAS FLAGRANCIA & 0 & 0 & 8 & 0 & 69 & 69 & ? & 0 & 67 & 2 \\
\hline POR ORDEN JUDICIAL & 0 & 0 & 8 & 0 & 7 & 7 & ? & 0 & 7 & 0 \\
\hline POR PORTE ILEGAL & 0 & 0 & 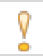 & 0 & 0 & 10 & $\mathscr{V}$ & 10 & 0 & 10 \\
\hline POR DECRETO & 0 & 0 & $?$ & 0 & 1 & 2 & 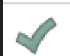 & 1 & 1 & 1 \\
\hline
\end{tabular}




\begin{tabular}{|c|c|c|c|c|c|c|c|c|c|c|}
\hline \multicolumn{11}{|c|}{ CUADRANTE 06} \\
\hline \multirow{2}{*}{ OPERATIVO } & \multicolumn{4}{|c|}{ SEMANA 52} & \multicolumn{4}{|c|}{ ACUMULADO } & \multicolumn{2}{|c|}{ LINEA BASE 2014} \\
\hline & 2014 & 2015 & & VAR & 2014 & 2015 & & VAR & 2014 & FALTA \\
\hline MERCANCIA RECUPERADA & 0 & 2 & $\mathscr{V}$ & 2 & 1 & 11 & $\mathscr{V}$ & 10 & 1 & 10 \\
\hline VEHICULOS RECUPERADO & 0 & 0 & ? & 0 & 0 & 0 & ? & 0 & 0 & 0 \\
\hline MOTOS RECUPERADA & 0 & 0 & 8 & 0 & 0 & 0 & ? & 0 & 0 & 0 \\
\hline ESTUPEFACIENTES & 0 & 0 & ? & 0 & 95 & 270 & $\mathscr{V}$ & 175 & 95 & 175 \\
\hline CAPTURAS FLAGRANCIA & 0 & 2 & $\mathscr{V}$ & 2 & 3 & 22 & $\mathscr{V}$ & 19 & 3 & 19 \\
\hline POR ORDEN JUDICIAL & 0 & 0 & 8 & 0 & 1 & 0 & $x$ & -1 & 1 & -1 \\
\hline POR PORTE ILEGAL & 0 & 0 & ? & 0 & 0 & 0 & ? & 0 & 0 & 0 \\
\hline POR DECRETO & 0 & 0 & 8 & 0 & 0 & 0 & 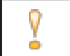 & 0 & 0 & 0 \\
\hline
\end{tabular}

\begin{tabular}{|c|c|c|c|c|c|c|c|c|c|c|}
\hline \multicolumn{11}{|c|}{ CUADRANTE 18} \\
\hline \multirow{2}{*}{ OPERATIVO } & \multicolumn{4}{|c|}{ SEMANA 52} & \multicolumn{4}{|c|}{ ACUMULADO } & \multicolumn{2}{|c|}{ LINEA BASE 2014} \\
\hline & 2014 & 2015 & & VAR & 2014 & 2015 & & VAR & 2014 & FALTA \\
\hline MERCANCIA RECUPERADA & 0 & 0 & 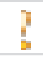 & 0 & 22 & 21 & th & -1 & 21 & 0 \\
\hline VEHICULOS RECUPERADO & 0 & 0 & 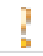 & 0 & 0 & 1 & 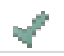 & 1 & 0 & 1 \\
\hline MOTOS RECUPERADA & 0 & 0 & 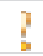 & 0 & 1 & 0 & H & -1 & 1 & -1 \\
\hline ESTUPEFACIENTES & 0 & 0 & 7 & 0 & 481 & 212 & th & -269 & 481 & -269 \\
\hline CAPTURAS FLAGRANCIA & 0 & 0 & 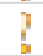 & 0 & 44 & 37 & At & -7 & 52 & -15 \\
\hline POR ORDEN JUDICIAL & 0 & 0 & 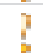 & 0 & 5 & 4 & th & -1 & 6 & -2 \\
\hline POR PORTE ILEGAL & 0 & 0 & 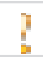 & 0 & 0 & 1 & 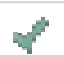 & 1 & 0 & 1 \\
\hline POR DECRETO & 0 & 0 & 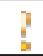 & 0 & 1 & 0 & H & -1 & 1 & -1 \\
\hline
\end{tabular}

\begin{tabular}{|c|c|c|c|c|c|c|c|c|c|c|}
\hline \multicolumn{11}{|c|}{ CUADRANTE 21} \\
\hline \multirow{2}{*}{ OPERATIVO } & \multicolumn{4}{|c|}{ SEMANA 52} & \multicolumn{4}{|c|}{ ACUMULADO } & \multicolumn{2}{|c|}{ LINEABASE 2014} \\
\hline & 2014 & 2015 & & VAR & 2014 & 2015 & & VAR & 2014 & FALTA \\
\hline MERCANCIA RECUPERADA & 0 & 0 & 8 & 0 & 21 & 17 & \multirow{2}{*}{$\frac{x}{?}$} & -4 & 2 & 15 \\
\hline VEHICULOS RECUPERADO & 0 & 0 & 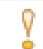 & 0 & 0 & 0 & & 0 & 0 & 0 \\
\hline MOTOS RECUPERADA & 0 & 0 & 8 & 0 & 0 & 0 & 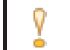 & 0 & 0 & 0 \\
\hline ESTUPEFACIENTES & 0 & 0 & 8 & 0 & 350 & 1018 & 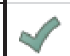 & 668 & 35 & 983 \\
\hline CAPTURAS FLAGRANCIA & 0 & 0 & 8 & 0 & 48 & 35 & 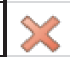 & -13 & 46 & -11 \\
\hline POR ORDEN JUDICIAL & 0 & 0 & $?$ & 0 & 2 & 9 & 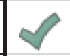 & 7 & 2 & 7 \\
\hline POR PORTE ILEGAL & 0 & 0 & ? & 0 & 0 & 0 & ? & 0 & 0 & 0 \\
\hline POR DECRETO & 0 & 0 & 8 & 0 & 0 & 0 & ? & 0 & 0 & 0 \\
\hline
\end{tabular}

Tabla 5. Tablas de Acciones Mínimas Requeridas (TAMIR) 2015.

Fuente: Hojas de registro de los cuadrantes 4, 5, 6, 18, 21 de Chapinero Central. 
En el año 2015 el operativo que más se realizó por todos los cuadrantes fue el de estupefacientes con una cifra de 8.053 lo que significa que para los cuadrantes en ese año la prioridad era incautar droga en el sector.

Teniendo en cuenta la cifra del total de operativos realizados en ese año es claro que en la zona de Chapinero Central se encuentra un índice muy alto de consumidores 0 vendedores de droga. Este fenómeno explicaría por qué es el barrio con más denuncias de hurto, precisamente porque algunos académicos y en especial el Departamento de Justicia de los Estados Unidos ha argumentado que las drogas están relacionadas con el crimen en múltiples formas. La mayoría de los casos se da una relación directa cuando el uso, posesión, manufactura o distribución de drogas clasifica a las personas como potenciales para cometer algún abuso. El principal argumento es que las drogas se relacionan con efectos comportamentales de las personas que tienden a generar violencia. De hecho, no solo son los efectos en las personas, sino que el tráfico de drogas genera violencia para tratar de controlar una zona donde los dealers manejan su distribución (Justice, 1994).

Lo interesante de la investigación es que desde el año 92, La Oficina Estadística de Justicia de los Estados Unidos tiene datos relacionados con drogas y crimen. Sin embargo, la mayoría de los casos reportados en ese entonces se debía al alcohol y en plano secundario bajo otras sustancias (Justice, 1994).

Si bien la investigación es realizada hace más de 20 años, se trae a colación porque Estados Unidos relaciona como droga al alcohol en sus investigaciones y con relación al hurto en Chapinero Central los operativos están encaminados netamente a estupefacientes, dejando de lado por supuesto al alcohol cuando se trata de disminuir el crimen.

\subsection{6: cuando los operativos con relación a estupefacientes disminuyeron, el hurto a personas también disminuyó}

\begin{tabular}{|c|c|c|c|c|c|c|c|c|c|c|}
\hline \multicolumn{11}{|c|}{ CUADRANTE 04} \\
\hline \multirow{2}{*}{ OPERATIVO } & \multicolumn{4}{|c|}{ SEMANA 52} & \multicolumn{4}{|c|}{ ACUMULADO } & \multicolumn{2}{|c|}{ LINEABASE 2015} \\
\hline & 2015 & 2016 & & VAR & 2015 & 2016 & & VAR & 2015 & FALTA \\
\hline MERCANCIA RECUPERADA & 0 & 0 & ? & 0 & 75 & 40 & 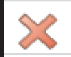 & -35 & 75 & -35 \\
\hline VEHICULOS RECUPERADO & 0 & 0 & ? & 0 & 0 & 0 & $\overline{8}$ & 0 & 0 & 0 \\
\hline MOTOS RECUPERADA & 0 & 0 & 8 & 0 & 0 & 0 & 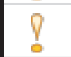 & 0 & 0 & 0 \\
\hline ESTUPEFACIENTES & 0 & 0 & $\overline{7}$ & 0 & 75 & 50 & x & -25 & 75 & -25 \\
\hline CAPTURAS FLAGRANCIA & 0 & 0 & ? & 0 & 99 & 52 & 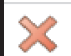 & -47 & 101 & -49 \\
\hline POR ORDEN JUDICIAL & 0 & 0 & ? & 0 & 0 & 4 & $\mathscr{O}$ & 4 & 2 & 2 \\
\hline POR PORTE ILEGAL & 0 & 0 & 8 & 0 & 0 & 0 & ? & 0 & 0 & 0 \\
\hline POR DECRETO & 0 & 0 & 7 & 0 & 0 & 1 & $\mathscr{V}$ & 1 & 0 & 1 \\
\hline
\end{tabular}




\begin{tabular}{|c|c|c|c|c|c|c|c|c|c|c|}
\hline \multicolumn{11}{|c|}{ CUADRANTE 05} \\
\hline \multirow{2}{*}{ OPERATIVO } & \multicolumn{4}{|c|}{ SEMANA 52} & \multicolumn{4}{|c|}{ ACUMULADO } & \multicolumn{2}{|c|}{ LINEABASE 2015} \\
\hline & 2015 & 2016 & & VAR & 2015 & 2016 & & VAR & 2015 & FALTA \\
\hline MERCANCIA RECUPERADA & 1 & 0 & \multirow{2}{*}{8} & -1 & 23 & 10 & \multirow{2}{*}{$\frac{1}{p}$} & -13 & 22 & -12 \\
\hline VEHICULOS RECUPERADO & 0 & 0 & & 0 & 0 & 0 & & 0 & 0 & 0 \\
\hline MOTOS RECUPERADA & 0 & 0 & ? & 0 & 6 & 2 & $x$ & -4 & 6 & -4 \\
\hline ESTUPEFACIENTES & 0 & 0 & 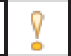 & 0 & 6477 & 571 & 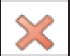 & -5906 & 6477 & -5906 \\
\hline CAPTURAS FLAGRANCIA & 1 & 0 & $\geqslant$ & -1 & 70 & 22 & 2 & -48 & 69 & -47 \\
\hline POR ORDEN JUDICIAL & 0 & 0 & ? & 0 & 7 & 5 & 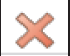 & -2 & 6 & -1 \\
\hline POR PORTE ILEGAL & 0 & 0 & $?$ & 0 & 10 & 0 & 2 & -10 & 10 & -10 \\
\hline POR DECRETO & 0 & 0 & 8 & 0 & 2 & 1 & 2 & -1 & 2 & -1 \\
\hline
\end{tabular}

\begin{tabular}{|c|c|c|c|c|c|c|c|c|c|c|}
\hline \multicolumn{11}{|c|}{ CUADRANTE 06} \\
\hline \multirow{2}{*}{ OPERATIVO } & \multicolumn{4}{|c|}{ SEMANA 52} & \multicolumn{4}{|c|}{ ACUMULADO } & \multicolumn{2}{|c|}{ LINEABASE 2015} \\
\hline & 2015 & 2016 & & VAR & 2015 & 2016 & & VAR & 2015 & FALTA \\
\hline MERCANCIA RECUPERADA & 2 & 0 & \multirow{2}{*}{$\frac{x}{?}$} & -2 & 13 & 13 & ! & 0 & 11 & 2 \\
\hline VEHICULOS RECUPERADO & 0 & 0 & & 0 & 0 & 0 & ! & 0 & 0 & 0 \\
\hline MOTOS RECUPERADA & 0 & 0 & ? & 0 & 0 & 0 & ? & 0 & 0 & 0 \\
\hline ESTUPEFACIENTES & 0 & 0 & ? & 0 & 270 & 115 & $\approx$ & -155 & 270 & -155 \\
\hline CAPTURAS FLAGRANCIA & 2 & 0 & $\times$ & -2 & 24 & 25 & 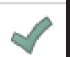 & 1 & 22 & 3 \\
\hline POR ORDEN JUDICIAL & 0 & 0 & ? & 0 & 0 & 0 & ? & 0 & 1 & -1 \\
\hline POR PORTE ILEGAL & 0 & 0 & ? & 0 & 0 & 0 & ? & 0 & 0 & 0 \\
\hline POR DECRETO & 0 & 0 & ? & 0 & 0 & 0 & ? & 0 & 0 & 0 \\
\hline
\end{tabular}

\begin{tabular}{|c|c|c|c|c|c|c|c|c|c|c|}
\hline \multicolumn{11}{|c|}{ CUADRANTE 18} \\
\hline \multirow{2}{*}{ OPERATIVO } & \multicolumn{4}{|c|}{ SEMANA 52} & \multicolumn{4}{|c|}{ ACUMULADO } & \multicolumn{2}{|c|}{ LINEABASE 2015} \\
\hline & 2015 & 2016 & & VAR & 2015 & 2016 & & VAR & 2015 & FALTA \\
\hline MERCANCIA RECUPERADA & 0 & 2 & 4 & 2 & 21 & 11 & th & -10 & 23 & -12 \\
\hline VEHICULOS RECUPERADO & 0 & 0 & ! & 0 & 1 & 0 & th & -1 & 1 & -1 \\
\hline MOTOS RECUPERADA & 0 & 0 & i & 0 & 0 & 0 & 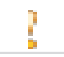 & 0 & 0 & 0 \\
\hline ESTUPEFACIENTES & 0 & 0 & $?$ & 0 & 212 & 12 & th & -200 & 212 & -200 \\
\hline CAPTURAS FLAGRANCIA & 0 & 2 & 4 & 2 & 37 & 20 & th & -17 & 39 & -19 \\
\hline POR ORDEN JUDICIAL & 0 & 0 & i & 0 & 4 & 0 & th & -4 & 1 & -1 \\
\hline POR PORTE ILEGAL & 0 & 0 & 7 & 0 & 1 & 0 & 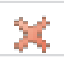 & -1 & 1 & -1 \\
\hline POR DECRETO & 0 & 0 & 7 & 0 & 0 & 0 & 7 & 0 & 0 & 0 \\
\hline
\end{tabular}




\begin{tabular}{|c|c|c|c|c|c|c|c|c|c|c|}
\hline \multicolumn{11}{|c|}{ CUADRANTE 21} \\
\hline \multirow{2}{*}{ OPERATIVO } & \multicolumn{4}{|c|}{ SEMANA 52} & \multicolumn{4}{|c|}{ ACUMULADO } & \multicolumn{2}{|c|}{ LINEABASE 2015} \\
\hline & 2015 & 2016 & & VAR & 2015 & 2016 & & VAR & 2015 & FALTA \\
\hline MERCANCIA RECUPERADA & 0 & 0 & $\pi$ & 0 & 17 & 5 & \multirow{2}{*}{ th } & -12 & 17 & -12 \\
\hline VEHICULOS RECUPERADO & 0 & 0 & ? & 0 & 0 & 0 & & 0 & 0 & 0 \\
\hline MOTOS RECUPERADA & 0 & 0 & 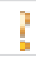 & 0 & 0 & 0 & 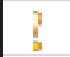 & 0 & 0 & 0 \\
\hline ESTUPEFACIENTES & 0 & 0 & 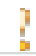 & 0 & 1018 & 645 & \multirow{4}{*}{ 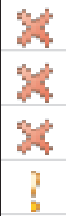 } & -373 & 1018 & -373 \\
\hline CAPTURAS FLAGRANCIA & 0 & 0 & 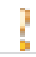 & 0 & 35 & 14 & & -21 & 36 & -22 \\
\hline POR ORDEN JUDICIAL & 0 & 0 & 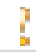 & 0 & 9 & 3 & & -6 & 13 & -10 \\
\hline POR PORTE ILEGAL & 0 & 0 & i & 0 & 0 & 0 & & 0 & 0 & 0 \\
\hline POR DECRETO & 0 & 0 & 7 & 0 & 0 & 0 & 7 & 0 & 0 & 0 \\
\hline
\end{tabular}

Tabla 6. Tablas de Acciones Mínimas Requeridas (TAMIR) 2016.

Fuente: Hojas de registro de los cuadrantes 4, 5, 6, 18, 21 de Chapinero Central.

Durante el año 2016 ocurre el suceso más interesante de esta investigación. La mayoría de los operativos nuevamente estuvieron relacionados con estupefacientes en todos los cuadrantes, con un total de 1.393 operativos. Sin embargo, en este año es cuando menos casos de hurto a personas se registraron en Chapinero Central con 466 en los datos de la Policía Metropolitana y con 358 en el total registrado por los cuadrantes de la zona.
Lo que llama la atención es que cuando los operativos con relación a estupefacientes disminuyeron, también disminuyó el hurto a personas. Por lo tanto, la relación entre el crimen y los estupefacientes en este caso no sería positiva y la estrategia de combatir el crimen con operativos encaminados en estos casos no estaría justificada porque cuando se disminuyen al parecer la seguridad en términos de hurto personas mejora.

9.3. 2017: la prioridad de los operativos sigue siendo los estupefacientes, aunque su relación con la disminución del crimen de hurto a personas no sea positiva

\begin{tabular}{|c|c|c|c|c|c|c|c|c|c|c|}
\hline \multicolumn{11}{|c|}{ CUADRANTE 04} \\
\hline \multirow{2}{*}{ OPERATIVO } & \multicolumn{4}{|c|}{ SEMANA 52} & \multicolumn{4}{|c|}{ ACUMULADO } & \multicolumn{2}{|c|}{ LINEA BASE 2016} \\
\hline & 2016 & 2017 & & VAR & 2016 & 2017 & & VAR & 2016 & FALTA \\
\hline MERCANCIA RECUPERADA & 1 & 2 & 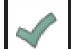 & 1 & 40 & 81 & $\mathscr{Q}$ & 41 & 40 & 41 \\
\hline VEHICULOS RECUPERADO & 0 & 0 & 7 & 0 & 1 & 0 & s & -1 & 0 & 0 \\
\hline MOTOS RECUPERADA & $\mathbf{0}$ & 0 & ? & 0 & 0 & $\mathbf{0}$ & ? & 0 & $\mathbf{0}$ & 0 \\
\hline ESTUPEFACIENTES & 0 & 0 & ? & 0 & 50 & 590 & $\vartheta$ & 540 & 50 & 540 \\
\hline CAPTURAS FLAGRANCIA & 1 & 2 & $\mathscr{V}$ & 1 & 52 & 99 & $\checkmark$ & 47 & 52 & 47 \\
\hline POR ORDEN JUDICIAL & $\mathbf{0}$ & 0 & 8 & 0 & 4 & 2 & x & -2 & 4 & -2 \\
\hline POR PORTE ILEGAL & $\mathbf{0}$ & 0 & 8 & 0 & $\mathbf{0}$ & 0 & 8 & 0 & $\mathbf{0}$ & 0 \\
\hline POR DECRETO & 0 & 0 & ఫ & 0 & 1 & 0 & $x$ & -1 & 1 & -1 \\
\hline
\end{tabular}




\begin{tabular}{|c|c|c|c|c|c|c|c|c|c|c|}
\hline \multicolumn{11}{|c|}{ CUADRANTE 05} \\
\hline \multirow{2}{*}{ OPERATIVO } & \multicolumn{4}{|c|}{ SEMANA 52} & \multicolumn{4}{|c|}{ ACUMULADO } & \multicolumn{2}{|c|}{ LINEABASE 2016} \\
\hline & 2016 & 2017 & & VAR & 2016 & 2017 & & VAR & 2016 & FALTA \\
\hline MERCANCIA RECUPERADA & 0 & 0 & ? & 0 & 10 & 14 & $\checkmark$ & 4 & 10 & 4 \\
\hline VEHICULOS RECUPERADO & 0 & 0 & $?$ & 0 & 0 & 0 & ? & 0 & 0 & 0 \\
\hline MOTOS RECUPERADA & 0 & 0 & $\overline{0}$ & 0 & 1 & 0 & \& & -1 & 2 & -2 \\
\hline ESTUPEFACIENTES & 0 & 0 & ? & 0 & 571 & 1760 & 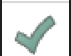 & 1189 & 571 & 1189 \\
\hline CAPTURAS FLAGRANCIA & 0 & 0 & ? & 0 & 22 & 35 & $\mathscr{V}$ & 13 & 22 & 13 \\
\hline POR ORDEN JUDICIAL & 0 & 0 & $!$ & 0 & 5 & 2 & $x$ & -3 & 5 & -3 \\
\hline POR PORTE ILEGAL & 0 & 0 & $?$ & 0 & 0 & 0 & ? & 0 & 0 & 0 \\
\hline POR DECRETO & 0 & 0 & ? & 0 & 1 & 0 & $x$ & -1 & 1 & -1 \\
\hline
\end{tabular}

\begin{tabular}{|c|c|c|c|c|c|c|c|c|c|c|}
\hline & & JA & & $\mathbf{M}$ & E 0 & & & & & \\
\hline חס्व & & $\overline{\text { EMAI }}$ & $\overline{A A}$ & & & $\overline{A C U M}$ & $\overline{J L A}$ & & LINEA & ISE 2016 \\
\hline UPEKAIIVO & 2016 & 2017 & & VAR & \begin{tabular}{|l|}
2016 \\
\end{tabular} & 2017 & & VAR & 2016 & FALTA \\
\hline MERCANCIA RECUPERADA & 0 & 0 & 8 & 0 & 13 & 4 & $x$ & -9 & 13 & -9 \\
\hline VEHICULOS RECUPERADO & 0 & 0 & Q & 0 & 0 & 0 & Q & 0 & 0 & 0 \\
\hline MOTOS RECUPERADA & 0 & 0 & ? & 0 & 1 & 1 & ? & 0 & 0 & 1 \\
\hline ESTUPEFACIENTES & 0 & 0 & 8 & 0 & 115 & 204 & $\mathscr{Q}$ & 89 & 115 & 89 \\
\hline CAPTURAS FLAGRANCIA & 0 & 0 & 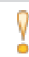 & 0 & 25 & 21 & $\mathrm{x}$ & -4 & 25 & -4 \\
\hline POR ORDEN JUDICIAL & 0 & 0 & 8 & 0 & 0 & 0 & ? & 0 & 0 & 0 \\
\hline POR PORTE ILEGAL & 0 & 0 & 8 & 0 & 0 & 4 & $\mathscr{Q}$ & 4 & 0 & 4 \\
\hline POR DECRETO & 0 & 0 & ? & 0 & 0 & 0 & ? & 0 & 0 & 0 \\
\hline
\end{tabular}

\begin{tabular}{|c|c|c|c|c|c|c|c|c|c|c|}
\hline \multicolumn{11}{|c|}{ CUADRANTE 18} \\
\hline \multirow{2}{*}{ OPERATIVO } & \multicolumn{4}{|c|}{ SEMANA 52} & \multicolumn{4}{|c|}{ ACUMULADO } & \multicolumn{2}{|c|}{ LINEABASE 2016} \\
\hline & 2016 & 2017 & & VAR & 2016 & 2017 & & VAR & 2016 & FALTA \\
\hline MERCANCIA RECUPERADA & 0 & 0 & 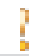 & 0 & 11 & 18 & 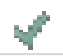 & 7 & 11 & 7 \\
\hline VEHICULOS RECUPERADO & 0 & 0 & : & 0 & 0 & 0 & 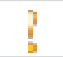 & 0 & 0 & 0 \\
\hline MOTOS RECUPERADA & 0 & 0 & t & 0 & 0 & 0 & 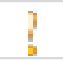 & 0 & 0 & 0 \\
\hline ESTUPEFACIENTES & 0 & 0 & : & 0 & 12 & 330 & 4 & 318 & 12 & 318 \\
\hline CAPTURAS FLAGRANCIA & 0 & 0 & $y$ & 0 & 20 & 22 & 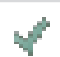 & 2 & 20 & 2 \\
\hline POR ORDEN JUDICIAL & 0 & 0 & $y$ & 0 & 0 & 1 & 4 & 1 & 0 & 1 \\
\hline POR PORTE ILEGAL & 0 & 0 & 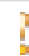 & 0 & 0 & 0 & 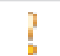 & 0 & 0 & 0 \\
\hline POR DECRETO & 0 & 0 & $y$ & 0 & 0 & 0 & 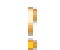 & 0 & 0 & 0 \\
\hline
\end{tabular}




\begin{tabular}{|c|c|c|c|c|c|c|c|c|c|c|}
\hline \multicolumn{11}{|c|}{ CUADRANTE 21} \\
\hline \multirow{2}{*}{ OPERATIVO } & \multicolumn{4}{|c|}{ SEMANA 52} & \multicolumn{4}{|c|}{ ACUMULADO } & \multicolumn{2}{|c|}{ LINEABASE 2016} \\
\hline & 2016 & 2017 & & VAR & 2016 & 2017 & & VAR & 2016 & FALTA \\
\hline MERCANCIA RECUPERADA & 0 & 0 & 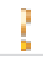 & 0 & 5 & 9 & 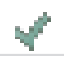 & 4 & 5 & 4 \\
\hline VEHICULOS RECUPERADO & 0 & 0 & 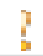 & 0 & 0 & 0 & 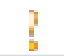 & 0 & 0 & 0 \\
\hline MOTOS RECUPERADA & 0 & 0 & 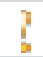 & 0 & 0 & 0 & ? & 0 & 0 & 0 \\
\hline ESTUPEFACIENTES & 0 & 0 & 7 & 0 & 645 & 342 & th & -303 & 645 & -303 \\
\hline CAPTURAS FLAGRANCIA & 0 & 0 & 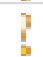 & 0 & 14 & 34 & $*$ & 20 & 14 & 20 \\
\hline POR ORDEN JUDICIAL & 0 & 0 & 7 & 0 & 3 & 2 & th & -1 & 3 & -1 \\
\hline POR PORTE ILEGAL & 0 & 0 & 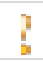 & 0 & 1 & 0 & 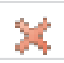 & -1 & 0 & 0 \\
\hline POR DECRETO & 0 & 0 & 7 & 0 & 0 & 0 & 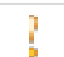 & 0 & 0 & 0 \\
\hline
\end{tabular}

Tabla 7. Tablas de Acciones Mínimas Requeridas (TAMIR) 2017.

Fuente: Hojas de registro de los cuadrantes 4, 5, 6, 18, 21 de Chapinero Central.

En el año 2017 los operativos de estupefacientes en los cuadrantes llegaron a un total de 3.226 que, si bien no es una cifra ni cercana a la del año 2015 , si permite analizar que para los cuadrantes la prioridad sigue siendo la incautación de droga, a pesar de que dichos operativos no traigan resultados positivos con respecto al delito que más afecta la percepción de inseguridad como lo es el hurto a personas.

En este año fue donde más se presenció un aumento de un año a otro con respecto a casos de hurto a personas tanto en los datos de la Policía (1271) como en el de los cuadrantes (805). Sin embargo, a penas, se realizaron 211 operativos relacionados con capturas en flagrancia.

El plan al parecer funciona, pero la política seguida por cada cuadrante con respecto a los estupefacientes no ha traído resultados positivos y cuando produjo resultados positivos, fue cuando menos operativos relacionados con drogas fueron realizados por los cuadrantes.

\subsection{8: la incautación de droga no mejoró y la seguridad ciudadana del barrio Chapinero Central en términos de hurto a personas}

\begin{tabular}{|c|c|c|c|c|c|c|c|c|c|c|}
\hline \multicolumn{11}{|c|}{ CUADRANTE 04} \\
\hline \multirow{2}{*}{ OPERATIVO } & \multicolumn{4}{|c|}{ SEM 52} & \multicolumn{4}{|c|}{ ACUMULADO } & \multicolumn{2}{|c|}{ LINEABASE 2017} \\
\hline & 2017 & 2018 & & VAR & 2017 & 2018 & & VAR & 2017 & FALTA \\
\hline MERCANCIA RECUPERADA & 1 & 3 & 8 & 2 & 82 & 136 & $\sqrt{ }$ & 54 & 81 & 55 \\
\hline VEHICULOS RECUPERADO & 0 & 0 & $\bar{p}$ & 0 & 0 & 0 & ? & 0 & 0 & 0 \\
\hline MOTOS RECUPERADA & 0 & 0 & D & 0 & 0 & 0 & ? & 0 & 0 & 0 \\
\hline ESTUPEFACIENTES & 0 & 0 & $\overline{0}$ & 0 & 590 & 460 & X & -130 & 59 & 401 \\
\hline CAPTURAS FLAGRANCIA & 1 & 3 & $\vartheta$ & 2 & 98 & 161 & $\vartheta$ & 63 & 99 & 62 \\
\hline POR ORDEN JUDICIAL & 0 & 0 & $\bar{l}$ & 0 & 2 & 2 & ? & 0 & 2 & 0 \\
\hline POR PORTE ILEGAL & 0 & 0 & ! & 0 & 0 & 1 & $\vartheta$ & 1 & 0 & 1 \\
\hline POR DECRETO & 0 & 0 & D & 0 & 0 & 0 & 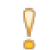 & 0 & 0 & 0 \\
\hline
\end{tabular}




\begin{tabular}{|c|c|c|c|c|c|c|c|c|c|c|}
\hline \multicolumn{11}{|c|}{ CUADRANTE 05} \\
\hline \multirow{2}{*}{ OPERATIVO } & \multicolumn{4}{|c|}{ SEM 52} & \multicolumn{4}{|c|}{ ACUMULADO } & \multicolumn{2}{|c|}{ LINEABASE 2017} \\
\hline & 2017 & 2018 & & VAR & 2017 & 2018 & & VAR & 2017 & FALTA \\
\hline MERCANCIA RECUPERADA & 0 & 0 & p & 0 & 15 & 20 & $\vartheta$ & 5 & 14 & 6 \\
\hline VEHICULOS RECUPERADO & 0 & 0 & ? & 0 & 0 & 0 & ? & 0 & 0 & 0 \\
\hline MOTOS RECUPERADA & 0 & 0 & ? & 0 & 0 & 0 & ? & 0 & 0 & 0 \\
\hline ESTUPEFACIENTES & 0 & 0 & ? & 0 & 1760 & 618,9 & $x$ & -1141 & 176 & 442,9 \\
\hline CAPTURAS FLAGRANCIA & 0 & 0 & ? & 0 & 35 & 40 & $\vartheta$ & 5 & 35 & 5 \\
\hline POR ORDEN JUDICIAL & 0 & 0 & p & 0 & 2 & 1 & $x$ & -1 & 2 & -1 \\
\hline POR PORTE ILEGAL & 0 & 0 & p & 0 & 0 & 1 & $\theta$ & 1 & 0 & 1 \\
\hline POR DECRETO & 0 & 0 & p & 0 & 0 & 0 & 0 & 0 & 0 & 0 \\
\hline
\end{tabular}

\begin{tabular}{|c|c|c|c|c|c|c|c|c|c|c|}
\hline \multicolumn{11}{|c|}{ CUADRANTE 06} \\
\hline \multirow{2}{*}{ OPERATIVO } & \multicolumn{4}{|c|}{ SEM 52} & \multicolumn{4}{|c|}{ ACUMULADO } & \multicolumn{2}{|c|}{ LINEABASE 2017} \\
\hline & 2017 & 2018 & & VAR & 2017 & 2018 & & VAR & 2017 & FALTA \\
\hline MERCANCIA RECUPERADA & 0 & 0 & ? & 0 & 4 & 10 & 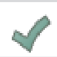 & 6 & 4 & 6 \\
\hline VEHICULOS RECUPERADO & 0 & 0 & ? & 0 & 0 & 0 & ? & 0 & 0 & 0 \\
\hline MOTOS RECUPERADA & 0 & 0 & ? & 0 & 1 & 0 & x & -1 & 1 & -1 \\
\hline ESTUPEFACIENTES & 0 & 0 & ! & 0 & 204 & 371,8 & $\mathscr{V}$ & 167,8 & 24 & 347,8 \\
\hline CAPTURAS FLAGRANCIA & 0 & 0 & ? & 0 & 21 & 24 & 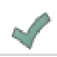 & 3 & 21 & 3 \\
\hline POR ORDEN JUDICIAL & 0 & 0 & ? & 0 & 0 & 2 & 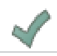 & 2 & 0 & 2 \\
\hline POR PORTE ILEGAL & 0 & 0 & ? & 0 & 4 & 1 & \& & -3 & 4 & -3 \\
\hline POR DECRETO & 0 & 0 & l & 0 & 0 & 0 & & 0 & 0 & 0 \\
\hline
\end{tabular}

\begin{tabular}{|c|c|c|c|c|c|c|c|c|c|c|}
\hline \multicolumn{11}{|c|}{ CUADRANTE 18} \\
\hline \multirow{2}{*}{ OPERATIVO } & \multicolumn{4}{|c|}{ SEM 52} & \multicolumn{4}{|c|}{ ACUMULADO } & \multicolumn{2}{|c|}{ LINEABASE 2017} \\
\hline & 2017 & 2018 & & VAR & 2017 & 2018 & & VAR & 2017 & FALTA \\
\hline MERCANCIA RECUPERADA & 0 & 1 & $y$ & 1 & 17 & 24 & 4 & 7 & 18 & 6 \\
\hline VEHICULOS RECUPERADO & 0 & 0 & 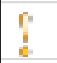 & 0 & 0 & 0 & 9 & 0 & 0 & 0 \\
\hline MOTOS RECUPERADA & 0 & 0 & $\mathrm{y}$ & 0 & 0 & 0 & 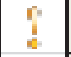 & 0 & 0 & 0 \\
\hline ESTUPEFACIENTES & 0 & 0 & i & 0 & 330 & 23,7 & 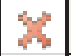 & $-306,3$ & 33 & $-9,3$ \\
\hline CAPTURAS FLAGRANCIA & 0 & 1 & $y$ & 1 & 22 & 33 & 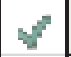 & 11 & 22 & 11 \\
\hline POR ORDEN JUDICIAL & 0 & 0 & i & 0 & 1 & 1 & 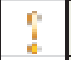 & 0 & 1 & 0 \\
\hline POR PORTE ILEGAL & 0 & 0 & is & 0 & 0 & 0 & 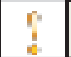 & 0 & 0 & 0 \\
\hline POR DECRETO & 0 & 0 & $\mathrm{i}$ & 0 & 0 & 0 & 8 & 0 & 0 & 0 \\
\hline
\end{tabular}




\begin{tabular}{|c|c|c|c|c|c|c|c|c|c|c|}
\hline \multicolumn{11}{|c|}{ CUADRANTE 21} \\
\hline \multirow{2}{*}{ OPERATIVO } & \multicolumn{4}{|c|}{ SEM 52} & \multicolumn{4}{|c|}{ ACUMULADO } & \multicolumn{2}{|c|}{ LINEABASE 2017} \\
\hline & 2017 & 2018 & & VAR & 2017 & 2018 & & VAR & 2017 & FALTA \\
\hline MERCANCIA RECUPERADA & 0 & 0 & in & 0 & 10 & 9 & \multirow{2}{*}{$\frac{1}{4}$} & -1 & 9 & 0 \\
\hline VEHICULOS RECUPERADO & 0 & 0 & 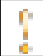 & 0 & 0 & 0 & & 0 & 0 & 0 \\
\hline MOTOS RECUPERADA & 0 & 0 & 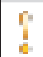 & 0 & 0 & 1 & 4 & 1 & 0 & 1 \\
\hline ESTUPEFACIENTES & 0 & 0 & Ii & 0 & 342 & 249,3 & th & $-92,7$ & 342 & $\begin{array}{l}-92,7 \\
\end{array}$ \\
\hline CAPTURAS FLAGRANCIA & 0 & 0 & in & 0 & 34 & 22 & th & -12 & 34 & -12 \\
\hline POR ORDEN JUDICIAL & 0 & 0 & 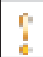 & 0 & 2 & 1 & H & -1 & 2 & -1 \\
\hline POR PORTE ILEGAL & 0 & 0 & 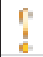 & 0 & 0 & 1 & 4 & 1 & 0 & 1 \\
\hline POR DECRETO & 0 & 0 & 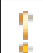 & 0 & 0 & 0 & I & 0 & 0 & 0 \\
\hline
\end{tabular}

Tabla 8. Tablas de Acciones Mínimas Requeridas (TAMIR) 2018.

Fuente: Hojas de registro de los cuadrantes 4, 5, 6, 18, 21 de Chapinero Central.

En el año 2018 nuevamente el enfoque de los cuadrantes estuvo relacionado con estupefacientes, aunque el número de operativos disminuyó con un total de 1.723 , sin embargo, se registró la cifra más alta hasta el momento en casos relacionados con hurto a personas en los cuadrantes con 1.371 casos y en la Policía Metropolitana con 1.715.

De lo anterior, se fue puede inferir que los operativos enfocados en estupefacientes no tienen ninguna relación con respecto al crimen de hurto a personas en Chapinero Central. Por lo cual, no hay una razón para que sea el principal operativo en la zona, pues su relación con la problemática que afecta al barrio no ha traído resultados positivos en los últimos 4 años.

Adicionalmente, es posible analizar que los operativos en orden de importancia con relación al mayor número de acciones realizadas están relacionados con: estupefacientes, capturas en flagrancia y mercancía recuperada.

Ahora bien, del análisis que se hace teniendo en cuenta los rubros más importantes del Plan Nacional de Vigilancia por Cuadrantes desde el 2015 hasta el 2018 en las zonas que ocupan la totalidad y un poco más del Barrio Chapinero Central, se puede resaltar lo siguiente:
1. Las hojas de registro funcionan en tanto que las cifras de los cuadrantes reflejan la problemática del barrio en general con respecto al hurto a personas. Cuando las estadísticas generales del barrio reflejan un aumento o disminución de casos, los datos que reportaban los cuadrantes también lo hacían.

2. Los principales problemas que enfrentan los cuadrantes están relacionados con hurto y con lesiones personales

3. Las Tablas TAMIR, están principalmente encaminadas a enfrentar el problema de estupefacientes, aunque en el diagnóstico no esté un rubro relacionado con drogas. Es decir, no hay una relación correspondiente a que en la zona se encuentre un alto índice de personas consumidoras o vendedoras de droga. Sin embargo, lo que sí se puede apreciar es que la mayoría de los operativos realizados por la policía de cada cuadrante está relacionada con los estupefacientes.

Se podría pensar que el fundamento detrás de esta decisión operativa de los cuadrantes tiene en cuenta las recomendaciones realizadas por el Departamento de Justicia de Estados Unidos y por estudios académicos que datan de los años 60s en que se encontró relación en Gran Bretaña entre el crimen y los consumidores de heroína. La relación 
básicamente está dada en que la mayoría de las personas que consumen alguna sustancia generalmente están involucradas en delitos (Bean, 2014). Sin embargo, en el caso de Chapinero Central, los operativos enfocados en estupefacientes no han logrado mitigar el hurto a personas en lazona.

4. Los operativos relacionados con capturas en flagrancia no han sido una herramienta eficaz para contrarrestar el alto índice de hurtos que enfrenta no sólo el barrio en general, sino cada cuadrante, porque la relación entre capturas en flagrancia e incluso por orden judicial no se acercan al número de hurtos que cada cuadrante enfrenta año a año.

5. En los operativos si bien no es posible determinar cuál ha sido la intención de cada cuadrante para enfrentar sus problemáticas, lo que sí se puede apreciar son los resultados de sus operativos. Por lo cual no es posible determinar si el objetivo de los policías de cada cuadrante ha sido enfocarse en estupefacientes porque si atacan la droga se disminuye el hurto, o si por el contrario se enfocan en las drogas porque es la problemática que más afecta el sector. Ahora bien, si este fuera el caso deberían tener un rubro relacionado con los estupefacientes en las hojas de registro donde se estipulan las problemáticas que enfrenta cada cuadrante.

Teniendo en cuenta lo anterior, si el delito de más afectación de todos los cuadrantes es el hurto a personas ¿por qué la mayoría de los operativos se concentraron en los estupefacientes? ¿Existe alguna relación entre mayor incautación de estupefacientes menor hurto de personas? Veamos.

\section{Principal problemática de cada cuadrante desde el 2015-2018: HURTO DE PERSONAS}

\begin{tabular}{|llllllll|}
\multicolumn{1}{c}{ Año } & $\begin{array}{c}\text { Cuadrante } \\
\mathbf{0 4}\end{array}$ & $\begin{array}{c}\text { Cuadrante } \\
\mathbf{0 5}\end{array}$ & $\begin{array}{c}\text { Cuadrante } \\
\mathbf{0 6}\end{array}$ & \multicolumn{2}{c}{$\begin{array}{c}\text { Cuadrante } \\
\mathbf{1 8}\end{array}$} & \multicolumn{2}{c|}{$\begin{array}{c}\text { Cuadrante } \\
\text { Total }\end{array}$} \\
\hline 2015 & 138 & 64 & 69 & 53 & 91 & 415 \\
\hline 2016 & 101 & 79 & 71 & 57 & 50 & 358 \\
\hline 2017 & 266 & 165 & 166 & 80 & 128 & 805 \\
\hline 2018 & 440 & 208 & 332 & 91 & 300 & 1371 \\
\hline TOTAL & 945 & 516 & 638 & 281 & 569 & 2949 \\
\hline
\end{tabular}

\section{Principal operativo realizado por cada cuadrante desde el 2015-2018: ESTUPEFACIENTES}

\begin{tabular}{|c|c|c|c|c|c|c|}
\hline Año & $\begin{array}{c}\text { Cuadrante } \\
04\end{array}$ & $\begin{array}{c}\text { Cuadrante } \\
05\end{array}$ & $\begin{array}{c}\text { Cuadrante } \\
06\end{array}$ & $\begin{array}{c}\text { Cuadrante } \\
18\end{array}$ & $\begin{array}{c}\text { Cuadrante } \\
21\end{array}$ & Total \\
\hline 2015 & 75 & 6477 & 270 & 212 & 1018 & 8052 \\
\hline 2016 & 50 & 571 & 115 & 12 & 645 & 1393 \\
\hline 2017 & 590 & 1760 & 204 & 330 & 342 & 3226 \\
\hline 2018 & 460 & 618,9 & 371,8 & 23,7 & 249,3 & 1723,7 \\
\hline TOTAL & 1175 & 9426,9 & 960,8 & 577,7 & 2254,3 & 14394,7 \\
\hline
\end{tabular}




\section{Relación entre operativo estupefacientes y hurto a personas en Chapinero Central:}

\begin{tabular}{|c|c|c|}
\hline Año & Hurto a personas & $\begin{array}{c}\text { Operativo } \\
\text { Estupefacientes }\end{array}$ \\
\hline 2015 & 415 & 8052 \\
\hline 2016 & 358 & 1393 \\
\hline 2017 & 805 & 3226 \\
\hline 2018 & 1371 & 1723,7 \\
\hline
\end{tabular}

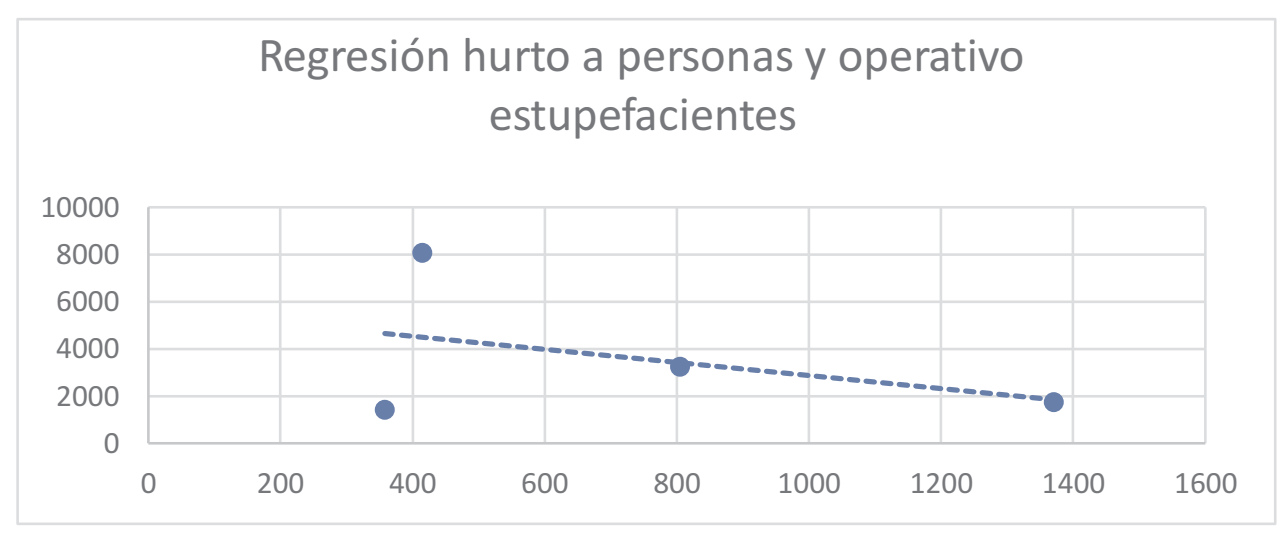

Coeficiente de Correlación -0,42085813

No existen datos suficientes para determinar si existe o no una correlación.

En este sentido, si bien la mayor problemática que enfrentan los cuadrantes que ayudan a determinar la situación del barrio Chapinero Central es el hurto de personas, y si la mayoría de los operativos están encaminados a incautar estupefacientes, no existe relación alguna entre los operativos y el hurto a personas. El impacto del Plan ha sido mínimo con relación a los delitos de mayor afectación para los ciudadanos, pues la estrategia operacional no ha logrado disminuir el mayor problema que enfrentan los ciudadanos del sector.

Asimismo, existen dos posibilidades correspondientes con el Plan Nacional de Vigilancia por Cuadrantes y su relación entre el hurto a personas y los estupefacientes. En primer lugar, es posible que para la Policía la mejor forma de combatir el hurto sea combatiendo la droga e incautando mercancía a consumidores o vendedores. En segundo lugar, es probable que los operativos estén enfocados en los estupefacientes porque sea el principal problema 0 queja de la comunidad de cada cuadrante. Ahora bien, en caso de que sea el principal problema debería estar en la hoja de registro, pero no se señala ese rubro como problema de los cuadrantes de Chapinero Central. Es decir, en cualquier situación el problema principal son los hurtos y como tal, los operativos no han estado encaminados 0 por lo menos no han logrado disminuir el número de hurtos en el barrio ni en cada cuadrante que ocupa la zona de Chapinero Central.

\section{CONCLUSIONES}

Por lo anterior, es posible concluir que el impacto del Plan Nacional de Vigilancia Comunitaria por Cuadrantes en el barrio Chapinero Central de Bogotá no ha sido positivo en los últimos 5 años, puesto que a pesar de que la estrategia operativa de dividir la ciudad por cuadrantes mejora el desplazamiento y la comprensión de zonas relativamente pequeñas junto con sus problemáticas, en Chapinero Central con los cuadrantes 04, 05, 06, 18 y 21 la relación entre el 
diagnóstico de cada cuadrante junto con los operativos no han generado una disminución significativa a la principal problemática del sector que es el hurto a personas.

En este sentido, no es posible afirmar que el plan no sea efectivo puesto que solamente se analizó un barrio en particular. Sin embargo, lo que sí es posible determinar es que los cuadrantes al enfocarse principalmente en incautaciones de estupefacientes no han podido disminuir el hurto a personas en Chapinero Central.

Asimismo, el segundo operativo con respecto las capturas en flagrancia realizadas por cada cuadrante del barrio tampoco han servido para disminuir el hurto a personas en la zona pues tiene tendencia a aumentar año tras año. Por lo cual, lo que es claro es que el plan a través de los cuadrantes con las hojas de registro si realiza una función certera sobre lo que está pasando en una escala focalizada en pequeñas zonas, pues todos los cuadrantes reflejaron que su principal problema era el hurto a personas, generando concordancia con los datos de la zona que ocupa el barrio completo con respecto a la situación de hurto a personas en Chapinero Central. Es decir que cada cuadrante hizo un diagnóstico particular que reflejaba exactamente el mismo problema en el diagnóstico general del barrio.

Los trabajos realizados anteriormente que han intentado evaluar el plan, lo han hecho con estadísticas muy generales sobre su funcionamiento. Por lo cual, al ser un plan meramente operativo que busca facilitarle a los policías la tarea de abarcar una ciudad completa a través de zonas más pequeñas que harían fácil el control sobre las principales problemáticas a través de la hoja de registro (diagnóstico) de cada zona de cada cuadrante, y a su vez luego de llevar las estadísticas de estas pequeñas zonas (cuadrantes) poder emplear el operativo concreto que ayude a disminuir o mitigar las problemáticas principales, se genera como expectativa que es un plan moderno y que tiene unas variaciones importantes con respecto al modelo de carabineros de Chile, de donde se tomó como base este Plan. Por ejemplo, como bien se mencionó anteriormente el modelo chileno fortalece la relación entre ciudadano y policía. Lo cual valdría la pena aprovechar esa característica para emplearla en el modelo colombiano. Sin embargo, como el objetivo del plan es dividir las zonas operativas de la policía en un rango mucho más pequeño para aumentar su eficacia, la mejor forma de evaluarlo es analizando un barrio en particular.

Es pertinente mencionar que, si bien el barrio Chapinero Central fue objeto de estudio en esta investigación por ser el barrio que más denuncias de hurto presentaba, también es importante mencionar que la mejor forma de evaluar el impacto de una estrategia de Seguridad Ciudadana como la del plan fue consiguiendo los mismos datos que ellos utilizan año a año desde el 2015, para evaluar la zona. Por lo cual, teniendo las hojas de registro y las tablas TAMIR, a pesar de que no se haga un trabajo estadístico profundo o complejo, las tablas permiten observar que en lo que respecta a los diagnósticos el trabajo está bien detallado, así como también en los operativos. El problema entonces es que el impacto deseado no se da, no porque el plan no funcione sino tal vez por la misma interpretación de los datos que la Policía misma le da.

En este sentido, valdría la pena analizar si las prioridades no estarían encaminadas a satisfacer las problemáticas de los ciudadanos, sino que por el contrario corresponden a un tema de reconocimiento de labor o de prestigio de la policía con relación a los operativos de estupefacientes y a las capturas en flagrancia; o si su labor está ligada con la relación entre drogas y crimen que ha sido investigada por académicos y por gobiernos como el de Estados Unidos.

Asimismo, es importante señalar que la zona de Chapinero Central pertenece a una localidad bastante difícil de controlar por la población flotante del sector que se caracteriza por tener 1.500 .000 de personas diarias, que sumados a los 166.000 generan un desafío en términos de seguridad 
ciudadana (Alcaldía de Chapinero, 2017). Es decir que, es bastante complejo para los policías del cuadrante controlar o prevenir los hurtos básicamente porque es físicamente imposible abarcar todas las zonas y con los recorridos disuasorios no se genera un gran impacto, ni tampoco con los operativos pues es claro que sigue siendo el barrio donde más denuncias por hurto se han generado en los últimos cinco años.

Por lo cual a modo de conclusión es claro que el impacto del Plan en Chapinero Central no ha sido positivo porque los hurtos a personas prevalecen y no se ha logrado mitigar. Ahora bien, el hecho de que no sea positivo no quiere decir que el Plan sea un fracaso, pues como se ha mencionado anteriormente tanto la Hoja de Registro como las Tablas TAMIR, han sido dos herramientas fundamentales para comprender las situaciones de cada cuadrante. Así pues, lo que valdría la pena repensar son las interpretaciones mismas que la Policía o el Gobierno le dan a los datos que ofrece el plan, puesto que las interpretaciones de estas estadísticas son las que permiten encaminar una política o estrategia dispuesta a mitigar los problemas de seguridad ciudadana en Bogotá. Sin embargo, puede que los objetivos del gobierno y de la policía se encarguen más de dar a conocer su trabajo con incautaciones de estupefacientes que en combatir las problemáticas de una manera más profunda.

Es posible entender que las problemáticas son bastante complejas con respecto a la relación entre el número de policías y los recursos, y ahí precisamente es donde yace el valor del Plan porque facilita operativamente la labor de los Policías. Sin embargo, se debería intentar a modo de recomendación imitar el modelo chileno no sólo se enfocó en mejorar la labor policial operativamente, sino que buscó estrechar los lazos entre los ciudadanos y la policía. De algún modo esto sería volver a la Policía Comunitaria, pero sería un intento que valdría la pena para que las problemáticas y los operativos sirvan en función de la seguridad ciudadana y de los principales problemas que afectan a los ciudadanos de la zona y no se dé espacio a otras interpretaciones de los datos que de alguna manera no generen un impacto positivo en la reducción de la criminalidad.

\section{REFERENCIAS BIBLIOGRÁFICAS}

I. Alcaldía de Chapinero. (2017). Infografía. Recuperado de www.chapinero.gov.co

II. Bean, P. (2014). Drugs and Crime (Fourth Edition ed.). New York: Routledge- Taylor \& Francis Group.

III. CCB. (2010). Observatorio de Seguridad en Bogotá. Bogotá.

IV. Ceballos., G. M. y. M. (2004). Bogotá: anomia de una transformación. Políticas de seguridad ciudadana 1995-2003: Editorial Pontificia Javeriana.

V. Díaz, C. (2011). El Modelo de Policía Comunitaria: El caso Chileno. Revista Chilena de Derecho y Ciencia Política, 2. doi: 10.7770 / rchdycp-V2N1-art37

VI. Frühling, H. (2007). The Impact of International Models of Policing in Latin America: The Case of Community Policing. Police Practice and Research, 8(2), 125-144. doi:10.1080/ 15614260701377638

VII. Frühling, H. (2012). A realistic look at Latin American community policing programmes. Policing and Society, 22(1), 76-88. doi:10.1080 /10439463.2011.636816

VIII. García, A. (2015). Definición y principales características del plan nacional de vigilancia comunitaria por cuadrantes -pnvcc- y del modelo nacional de vigilancia comunitaria por cuadrantes en la policia nacional de colombia. (Especialización Administración de la Seguridad). Universidad Militar Nueva Granada, Bogotá, Colombia.

IX. Justice, U. S. D. O. (1994). Fact Sheet: DrugRelated Crime. Retrieved from https://www. bjs.gov/content/pub/pdf/DRRC.PDF

X. Nación, F. G. D. L. (2018). El sistema de denuncia virtual 'Adenunciar' crece. Bogotá Recuperado de https://www.fiscalia.gov.co/ colombia/fiscal-general-de-la-nacion/elsistema-de-denuncia-virtual-adenunciarcrecel 
XI. OEA. (2013). "Premio Interamericano a la Innovación para la Gestión Pública Efectiva". Recuperado de https://www.oas.org/es/ sap/dgpe/innovacion/banco/PLAN\%20NACI ONAL\%20DE\%20VIGILANCIA\%20COMUNI TARIA\%20POR\%20CUADRANTES.pdf

XII. Sandoval-Garrido, L., y Marin, M. (2017). Efecto de la red de comunicación de cuadrantes de la policía en las tasas de criminalidad en Bogotá, Colombia. Ecos de Economía, 21, 4-23. doi:10.17230/ecos.2017. 45.1

XIII. Socha, D. (2013). Plan Nacional de Vigilancia Comunitaria por Cuadrantes: Un análisis al modelo de Policía en Bogotá desde la Epistemología del Sur. (Maestría en Estudios Políticos). Universidad Nacional de Colombia, Bogotá.

XIV. Vásquez, J. C. R. (2012). Community police in Colombia: an idle process. Policing and Society, 22(1), 43-56. doi:10.1080/10439463. 2011.597855 